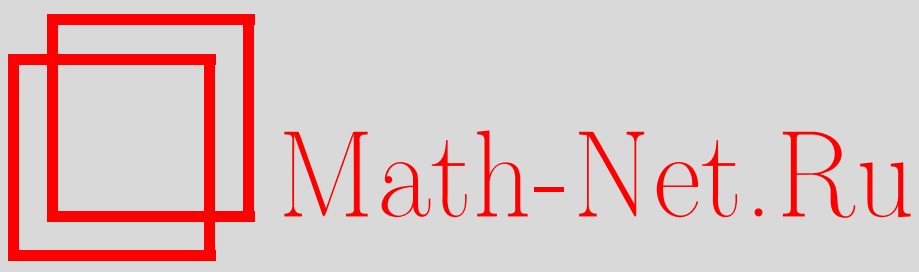

А. А. Толстоногов, О решениях эволюционной управляемой системы, зависящей от параметров, Матем. сб., 2003, том 194, номер 9, 113-140

DOI: https://doi.org/10.4213/sm769

Использование Общероссийского математического портала Math-Net.Ru подразумевает, что вы прочитали и согласны с пользовательским соглашением

http: //www. mathnet.ru/rus/agreement

Параметры загрузки:

IP: 3.85 .5 .30

26 апреля 2023 г., 11:23:45 


\title{
А. А. Толстоногов \\ О решениях эволюционной управляемой системы, зависящей от параметров
}

\begin{abstract}
Изучается управляемая система, описьваемая нелинейньм эволюционньм уравнением первого порядка со смешанными невьпукльми ограничениями на управление. Система зависит от параметров. Параметры входят во все данные системы, включая нелинейный эволюционный оператор и ограничения на управление. Также рассматривается система с овьпукленными ограничениями на управление. $\mathrm{C}$ помощью общего понятия $G$-сходимости операторов доказьвается существование непрерывных относительно параметров селекторов, значениями которых являются решения исходной системы, и непрерывная версия теоремы релаксации для таких селекторов. Последнее означает аппроксимацию непрерывных селекторов - решений системы с овыпукленньми ограничениями непрерывньпи селекторами - решениями исходной системы. Рассматривается пример управляемой параболической системы.
\end{abstract}

Библиографиия: 24 названия.

\section{§ 1. Введение}

В работе изучается зависимость от параметров множества допустимых пар “траектория-управление" нелинейной эволюционной управляемой системы первого порядка со смешанными невыпуклыми ограничениями на управление. При этом параметры входят в начальные условия, эволюционные операторы и в ограничения на управление. Наря ду с исходной системой рассматривается система с овыпукленными ограничениями на управление. Основное внимание в работе уделено существованию непрерывных селекторов многозначных отображений, которые определены на пространстве параметров и значениями которых являются множества решений управляемых систем. Рассматриваются также вопросы аппроксимации непрерывных селекторов (теоремы релаксации для селекторов) управляемой системы с овыпукленными ограничениями подобными селекторами исходной системы. Помимо этих вопросов изучается ряд топологических свойств множества решений, таких как абсолютная ретрактность, дугообразная связность и т. д. Так как значениями указанных выше отображений являются невьпуклые множества, то в работе выделен класс многозначных отображений с невыпуклыми значениями, у которых существуют непрерывные селекторы, что является актуальной современной проблемой (см. [1]). Необходимость рассмотрения подобных вопросов обусловлена также различными приложениями. Среди

Работа выполнена при финансовой поддержке Российского фонда фундаментальных исследований (грант № 03-01-00203), Российского фонда фундаменталњных исследований Государственного фонда естественных наук Китая (грант № 02-01-39006) и фонда INTAS (грант № 2000-0015). 
них следует отметить задачи идентификации и параметрической оптимизации (см. [2]).

Вопросы существования непрерывных селекторов, значениями которых являются решения обыкновенных дифференциальных включений, изучались многими авторами. Наиболее общие в этом направлении результаты получены в [3]. Подобные вопросы для включений типа Гурса-Дарбу и некоторых классов эволюционных включений рассматривались в работах [4]-[7] и др.

Что же касается релаксационных теорем для селекторов, то для обыкновенных дифференциальных включений они рассматривались в [8]. В этой работе роль параметра играли начальные условия. Общий случай, когда параметр входит и в правую часть обыкновенного дифференциального включения, изучен в [3]. Для линейных эволюционных включений, у которых линейные, плотно определенные операторы и правые части зависят от параметра, релаксационные теоремы для селекторов доказаны в [7].

Отличие нашей работы от известных состоит в следуюшем. Мы рассматриваем нелинейные управляемые системы, у которых параметры входят как в начальные условия, так и в нелинейные эволюционные операторы и в смешанные невыпуклые ограничения на управление. Затронутые в работе вопросы в такой постановке ранее не изучались не только для нелинейных эволюционных управляемых систем, но и для эволюционных включений. Более того, управляемые системы являются более сложным объектом с точки зрения изучения свойств решений, чем включения. Это объясняется в первую очередь тем, что реакция траектории на изменение управления носит более сложньй характер, чем реакция траектории на изменение ее производной у включения.

При изучении указанных выше вопросов мы развиваем подходы, отличные от традиционных (см. [4], [5], [8] и др.). Нашим основным инструментом является теория $G$-сходимости эволюционных операторов [9]-[11] и др. и теория непрерывных селекторов, проходящих через неподвижные точки многозначных отображений с невьпуклыми разложимыми значениями, зависящими от параметра, развитая в работах автора [6], [12], [13].

Мы рассматриваем следующую нелинейную эволюционную управляемую систему.

Пусть $T=[0,1], H$ - сепарабельное гильбертово пространство, $V$ - сепарабельное рефилксивное банахово пространство, которое плотно, непрерьвно и компактно вложено в $H$. Отождествляя $H$ с его топологически сопряженным, мы имеем $V \hookrightarrow H \hookrightarrow V^{*}$, где $V^{*}$ - пространство, топологически сопряженное к $V$. При этом все вложения являются непрерывными, плотньми и компактными. Такая тройка пространств обычно называется эволюиионной. Пусть $Y$ - сепарабельное рефлексивное банахово пространство, моделируюшее пространство управлений, и $\Lambda$ - сепарабельное метрическое пространство значений параметра. Управляемая система описывается следуюшим эволюционным уравнением:

$$
\begin{gathered}
\dot{x}(t)+A(t, x(t), \xi)=B(t) u(t) \quad \text { почти всюду }, \\
x(0)=\lambda \in H, \quad \xi \in \Lambda,
\end{gathered}
$$

со смешанными ограничениями на управление

$$
u(t) \in U(t, x(t), \xi) \quad \text { почти всюду, }
$$


где $A: T \times V \times \Lambda \rightarrow V^{*}$ - нелинейный оператор, $U: T \times H \times \Lambda \rightarrow Y$ - многозначное отображение с невыпуклыми замкнутьми значениями, $B(t)$ при почти каждом $t \in T$ - линейный непрерывный оператор из $Y$ в $H$. Наряду с ограничениями (1.2) мы рассматриваем следующее ограничение:

$$
u(t) \in \overline{\mathrm{co}} U(t, x(t), \xi) \quad \text { почти всюду, }
$$

где со означает замкнутую выпуклую оболочку множества.

При фиксированных $\xi \in \Lambda, \lambda \in H$ под решением управляемой системы (1.1), $(1.2)$ мы понимаем пару $(x(\xi, \lambda), u(\xi, \lambda))$, состоящую из траектории $x(\xi, \lambda)$ и управления $u(\xi, \lambda)$, удовлетворяющих уравнению (1.1) и ограничению (1.2). Основная цель работы - изучение свойств пар $(x(\xi, \lambda), u(\xi, \lambda))$ и их зависимости от параметров $(\xi, \lambda)$.

Работа состоит из семи параграфов.

В $\S 1$ дается краткий экскурс в состояние проблемы и ее постановка.

В $\S 2$ приводятся основные обозначения, определения и предположения.

В $\S 3$ приведены доказательства ряда вспомогательных результатов, необходимых в дальнейшем.

В $\S 4$ изучаются свойства многозначного оператора Немыцкого, порожденного управляемой системой.

В $\S 5$ изучаются свойства неподвижных точек многозначного оператора Немьщкого и их зависимость от параметров.

В $\S 6$ изложены основные результаты работы.

В $\S 7$ рассматривается пример управляемой параболической системы и применительно к этой системе дается конкретизация абстрактных результатов из $\S 6$.

\section{§ 2. Основные определения и обозначения}

Пусть $(X, d)$ - метрическое пространство, $T=[0,1]$ - отрезок числовой полупрямой $\mathbb{R}^{+}$с мерой Лебега $\mu$ и с $\sigma$-алгеброй $\Sigma \mu$-измеримых подмножеств из $T$.

Всюду в дальнейшем мы используем следующие обозначения: $c X-$ семейство всех непустых, замкнутых подмножеств из $X, \operatorname{cb} X$ - семейство всех непустых, замкнутых, ограниченных подмножеств из $X$. На пространстве с $X$ определим обобщенную метрику Хаусдорфа

$$
D_{X}(A, B)=\max \left\{\sup _{a \in A} \inf _{b \in B} d(a, b), \sup _{b \in B} \inf _{a \in A} d(a, b)\right\}
$$

Поскольку функция $D_{X}(\cdot, \cdot)$ может принимать бесконечные значения, то она называется обобщенной метрикой.

Пусть $Y$ - некоторое топологическое пространство. Многозначное отображение $F: Y \rightarrow c X$ называется непрерьвным, если оно является непрерывным из $Y$ в $\left(\mathrm{c} X, D_{X}(\cdot, \cdot)\right)$.

Многозначное отображение $F: X \rightarrow Y$ называется полунепрерывным снизу по Bветорису, если для любого открытого множества $E \subset Y$ множество $F^{-1}(E)=$ $\{x \in X: F(x) \cap E \neq \varnothing\}$ открыто.

Многозначное отображение $F: X \rightarrow Y$ называется полунепрерывным сверху nо Вветорису, если для любого открытого множества $E \subset Y$ множество $F^{+}(E)=$ $\{x \in X: F(x) \subset E\}$ открыто. 
Многозначное отображение $F: X \rightarrow Y$ назьвается непрерывнылм по Bветоpucy, если оно одновременно полунепрерывно снизу и сверху по Вьеторису.

Если отображение $F: Y \rightarrow c X$ непрерьвно, то оно полунепрерывно снизу по Вьеторису и имеет замкнутьй график.

Отметим, что если $Y$ - метрическое пространство, то определение полунепрерывности снизу по Вьеторису эквивалентно следующему: для любых $x \in X$, $y \in F(x)$ и для любой последовательности $x_{n} \rightarrow x$ существует последовательность $y_{n} \in F\left(x_{n}\right), n \geqslant 1$, сходяшаяся к $y$.

Многозначное отображение $F: T \rightarrow \mathrm{c} X$ называется измеримым, если $F^{-1}(E)=\{t \in T: F(t) \cap E \neq \varnothing\} \in \Sigma$ для любого замкнутого множества $E \subset X$.

Подмножество $A$ хаусдорфова топологического пространства $Y$ называется ретрактом, если существует непрерывное отображение $g: Y \rightarrow A$ такое, что $g(y)=y, y \in A$. Любое такое отображение $g$ мы называем ретракиией $Y$ на $A$.

Множество $A \subset Y$ называется абсолютным ретрактом, если для любого сепарабельного метрического пространства $Z$, для любого замкнутого множества $B \subset Z$ и для любого непрерывного отображения $g: B \rightarrow A$ сушествует непрерывное продолжение $g$ на $Z$ со значениями в $A[14]$.

Множество $A$ называется дугообразно связным, если любую пару его точек можно соединить дугой [14].

Множество $A$ называется линейно связным, если для любых его точек $y_{1}, y_{2}$ существует непрерывное отображение $g:[0,1] \rightarrow A$ такое, что $g(0)=y_{1}, g(1)=y_{2}$ $[15]$.

Известно, что для хаусдорфова пространства $Y$ понятия дугообразной и линейной связности множества $A \subset Y$ совпадают [15].

Множество $K$ измеримых функций из $T$ в $X$ называется разложсимым, если для любых $u, v \in K, E \in \Sigma$ элемент $\chi(E) u+\chi(T \backslash E) v$ принадлежит $K$, где $\chi(E)-$ характеристическая функция множества $E$.

Пусть $\left(Z,\|\cdot\|_{Z}\right)$ - банахово пространство. Множество $K \subset L^{p}(T, Z), 1 \leqslant p<\infty$, называется равномерно р-интегрируемым, если для любого $\varepsilon>0$ существует $\delta(\varepsilon)>0$ такое, что

$$
\int_{E}\|f(t)\|_{Z}^{p} d t<\varepsilon
$$

для любого $E \in \Sigma, \mu(E) \leqslant \delta(\varepsilon)$, и всех $f \in K$.

Всюду в дальнейшем мы используем следуюшие фиксированные обозначения. Пусть $\left(V, H, V^{*}\right)$ - эволюционная тройка пространств. Через $\|\cdot\|$ (соответственно $|\cdot|_{H},\|\cdot\|_{*}$ ) мы обозначаем норму пространства $V$ (соответственно $H, V^{*}$ ). Под $\left\langle x, x^{\prime}\right\rangle, x \in V, x^{\prime} \in V^{*}$, мы понимаем каноническую билинейную форму, устанавливающую двойственность между $V$ и $V^{*}$, а $(\cdot, \cdot)$ означает скалярное произведение в $H$. Как обычно, мы считаем, что форома $\langle\cdot, \cdot\rangle$ и скалярное произведение $(\cdot, \cdot)$ согласованы, т.е. сужение $\langle\cdot, \cdot\rangle$ на $V \times H$ совпадает с $(\cdot, \cdot)$.

Всюду в дальнейшем число $p$ удовлетворяет условию $2 \leqslant p<\infty$. Мы введем следующие пространства:

$$
\mathscr{V}=L^{p}(T, V), \quad \mathscr{H}=L^{p}(T, H), \quad \mathscr{H}^{*}=L^{q}(T, H), \quad \mathscr{V}^{*}=L^{q}\left(T, V^{*}\right), \quad \frac{1}{p}+\frac{1}{q}=1,
$$

и пространство $\mathscr{W}=\left\{\nu \in \mathscr{V}: \dot{\nu} \in \mathscr{V}^{*}\right\}$ с нормой $\|\nu\|_{\mathscr{W}}=\left(\|\nu\|_{\mathscr{V}}^{2}+\|\dot{\nu}\|_{\mathscr{V} *}^{2}\right)^{1 / 2}$, где производная понимается в смысле векторных распределений. Пространство $\mathscr{W}$ 
с нормой $\|\cdot\| \mathscr{w}$ является сепарабельньм, рефлексивньм, банаховым пространством. Известно, что любой элемент из $\mathscr{W}$ можно отождествить с элементом из $C(T, H)$ путем изменения его значений на множестве нулевой меры и вложение $\mathscr{W} \hookrightarrow C(T, H)$ непрерывно, а вложение $\mathscr{W} \hookrightarrow \mathscr{H}$ компактно.

Билинейную форму, устанавливающую двойственность между $\mathscr{H}$ и $\mathscr{H}^{*}$, мы будем обозначать $(\cdot, \cdot) \mathscr{H}$. Она имеет вид

$$
\left(y, y^{\prime}\right)_{\mathscr{H}}=\int_{T}\left(y(t), y^{\prime}(t)\right) d t, \quad y \in \mathscr{H}, \quad y^{\prime} \in \mathscr{H}^{*} .
$$

Для любого банахова пространства $X$ символ $w$ - $X$ означает, что пространство $X$ наделено слабой $\sigma\left(X, X^{*}\right)$ топологией [16]. Такое же обозначение мы используем и для подмножеств из $X$. Во всех остальных случаях мы считаем, что пространство $X$ и его подмножества наделены сильной (нормированной) топологией.

Пусть $A_{n}, A: V \rightarrow V^{*}, n \geqslant 1,-$ последовательность операторов. Будем говорить, что последовательность $A_{n}, n \geqslant 1, G$-сходится к $A$, если сушествуют обратные операторы $A_{n}^{-1}, A^{-1}: V^{*} \rightarrow V, n \geqslant 1$, и для любого $v^{\prime} \in V^{*}$ последовательность $A_{n}^{-1} v^{\prime} \rightarrow A^{-1} v^{\prime}$ в $w-V$ (и, следовательно, в $H$ ) [9], [11].

Решением управляемой системы (1.1), (1.2) при фиксированных $\xi \in \Lambda, \lambda \in H$ мы называем пару $(x(\xi, \lambda), u(\xi, \lambda))$, состоящую из траектории $x(\xi, \lambda) \in \mathscr{W} \hookrightarrow$ $C(T, H)$ и управления $u(\xi, \lambda) \in L^{q}(T, Y)$, удовлетворяющих почти всюду уравнению (1.1) и ограничению (1.2). Аналогично определяется решение управляемой системы (1.1), (1.3). Через $\mathscr{R}_{U}(\xi, \lambda) \subset \mathscr{W} \times L^{q}(T, Y) \hookrightarrow C(T, H) \times L^{q}(T, Y)$ мы обозначаем множество всех решений управляемой системы (1.1), (1.2). Множество всех решений управляемой системы (1.1), (1.3) обозначается через $\mathscr{R} \overline{\operatorname{co}} U(\xi, \lambda)$.

Введем следующие предположения (гипотезы).

Гипотезы Г $(A)$. Считаем, что $A: T \times V \times \Lambda \rightarrow V^{*}$ является оператором таким, что:

1) отображение $x \mapsto A(t, x, \xi)$ радиально непрерывно, т.е. для любых $x, y \in V$ отображение $\beta \mapsto\langle y, A(t, x+\beta y, \xi)\rangle$ является непрерывным из $[0,1]$ в $\mathbb{R}$ при любом $\xi \in \Lambda$;

2) пусть $\Theta_{V}, \Theta_{V^{*}}$ - нулевые элементы пространств $V$ и $V^{*}$, тогда

$$
A\left(t, \Theta_{V}, \xi\right)=\Theta_{V^{*}} \text { для всех }(t, \xi) \in T \times \Lambda ;
$$

3) для компактного множества $K \subset \Lambda$ существует $m_{K}>0$ такое, что

$$
m_{K}\|x-y\|^{p} \leqslant\langle x-y, A(t, x, \xi)-A(t, y, \xi)\rangle,
$$

$x, y \in V, t \in T, \xi \in K$ (строгая монотонность);

4) для компактного множества $K \subset \Lambda$ сушествует $M_{K}>0$ такое, что

$$
\|A(t, x, \xi)\|_{*} \leqslant M_{K}\left(1+\|x\|^{p-1}\right)
$$

$x \in V, t \in T, \xi \in K$

5) существует неубывающая функция $c_{K}(\tau), c_{K}(\tau) \downarrow 0$ при $\tau \rightarrow 0+$, такая, что

$$
\|A(t, x, \xi)-A(t+\tau, x, \xi)\|_{*} \leqslant c_{K}(\tau)\left(1+\|x\|^{p-1}\right),
$$

$t, t+\tau \in T, x \in V, \xi \in K$

$6)$ если $\xi_{n} \rightarrow \xi$, то последовательность операторов $A\left(t, \cdot, \xi_{n}\right), n \geqslant 1, G$-сходится к $A(t, \cdot, \xi), t \in T$. 
Всюду в дальнейшем, не оговаривая особо, считаем, что гипотезы $\Gamma(A)$ имеют место.

ГиПотезЫ $\Gamma(B)$. Считаем, что $B: T \rightarrow \mathscr{L}(Y, H)$, где $\mathscr{L}(Y, H)$ - пространство непрерывных линейных операторов из $Y$ в $H$, является отображением таким, что

1) отображение $t \mapsto B(t) u$ измеримо для любого $u \in Y$;

2) справедливо

$$
\|B(t)\|_{\mathscr{L}(Y, H)} \leqslant m, \quad m>0, \quad \text { п.в. }
$$

Гипотезы $\Gamma(U)$. Считаем, что $U: T \times H \times \Lambda \rightarrow$ сY является многозначным отображением таким, что:

1) отображение $t \mapsto U(t, x, \xi)$ измеримо, $x \in H, \xi \in \Lambda$;

2) при почти всех $t \in T$ и любом $x \in H$ отображение $\xi \mapsto U(t, x, \xi)$ непрерыв$\mathrm{HO}$;

3) существует непрерывная функция $l: \Lambda \rightarrow L^{q}\left(T, \mathbb{R}^{+}\right)$такая, что для любых $x, y \in H, \xi \in \Lambda$ почти всюду

$$
D_{Y}(U(t, x, \xi), U(t, y, \xi)) \leqslant l(\xi)(t)|x-y|_{H}
$$

4) для любой сходящейся последовательности $\xi_{n} \in \Lambda, n \geqslant 1$, последовательность числовых функций $d_{Y}\left(\Theta_{Y}, U\left(t, \Theta_{H}, \xi_{n}\right)\right), n \geqslant 1$, равномерно $q$-интегрируема, где $d_{Y}\left(\Theta_{Y}, U\left(t, \Theta_{H}, \xi_{n}\right)\right)$ - расстояние от нулевого элемента $\Theta_{Y}$ пространства $Y$ до множества $U\left(t, \Theta_{H}, \xi_{n}\right)$, а $\Theta_{H}$ - нулевой элемент пространства $H$.

Всюду в дальнейшем, если не оговорено противное, мы считаем, что гипотезы $\Gamma(U)$ 1)-4) имеют место.

ЗАмЕчАнИЕ 2.1. Из (2.1), (2.2) следует, что

$$
m_{K}\|x\|^{p} \leqslant\langle x, A(t, x, \xi)\rangle
$$

для всех $t \in T, x \in V, \xi \in K$, т.е. для любых $t \in T, \xi \in \Lambda$ оператор $A(t, \cdot, \xi)$ является коэрцитивным. Поэтому согласно [17; гл. III, $\S 2$, теорема 2.2] для любых $t \in T, \xi \in \Lambda$ сушествует обратный оператор $A^{-1}(t, \cdot, \xi)$. Это означает, что гипотеза $\Gamma(A) 6)$ имеет смысл.

ЗАМЕЧАНИЕ 2.2. Гипотезы $Г(A) 1)-4), \Gamma(B)$ являются традиционньми, при которых для фиксированного $\xi$ эволюционное уравнение (1.1) имеет единственное решение (см., например, [17; гл. VI, $\S 1$ теорема 1.1 и примеры]). Согласно [11; лемма 5] гипотезы $\Gamma(A)$ обеспечивают выполнение более общих предположений $\mathrm{A})-\mathrm{D})$ [11], при которых справедлива теорема 1 из [11] (ее мы будем использовать в дальнейшем).

ЗАмечАниЕ 2.3. Из гипотезы $\Gamma(U) 1)$ следует, что при любом $\xi \in \Lambda$ функция $t \mapsto d_{Y}\left(\Theta_{Y}, U\left(t, \Theta_{H}, \xi\right)\right)$ измерима. Поэтому гипотеза $\left.\Gamma(U) 4\right)$ будет иметь место, если для любого компакта $K \subset \Lambda$ сушествуют функции $a_{K}, b_{K} \in L^{q}\left(T, \mathbb{R}^{+}\right)$такие, что почти всюду для любых $x \in H, \xi \in K$

$$
\|U(t, x, \xi)\|_{Y} \leqslant a_{K}(t)+b_{K}(t)|x|_{H}^{2 / q},
$$

где $\|U(t, x, \xi)\|_{Y}=\sup \left\{\|u\|_{Y}: u \in U(t, x, \xi)\right\}$. 


\section{§ 3. Вспомогательные результаты}

В дальнейшем нам понадобятся априорные оценки для траекторий управляемой системы (1.1), (1.3) и, следовательно, системы (1.1), (1.2).

Лемма 3.1. Пусть $K \subset \Lambda$ - компактное множество, $D \subset H$ - ограниченное множество и выполняется неравенство (2.6). Тогда существуют $M_{i}>0, i=1, \ldots, 4$, такие, что для любой траектории $x(\xi, \lambda) \in \mathscr{W}, \xi \in K$, $\lambda \in D$, управляемой системы (1.1), (1.3) имеют место неравенства

$$
\begin{gathered}
|x(\xi, \lambda)(t)|_{H} \leqslant M_{1}, \quad t \in T \\
\|x(\xi, \lambda)\|_{\mathscr{V}} \leqslant M_{2}, \quad\|\dot{x}(\xi, \lambda)\|_{\mathscr{V} *} \leqslant M_{3}, \\
\|x(\xi, \lambda)\|_{\mathscr{W}} \leqslant M_{4} .
\end{gathered}
$$

Доказательство оценок (3.1)-(3.3) проводится по достаточно известным схемам (см., например, [18] и др.), и мы его опускаем.

Через $d_{L}(v, B)$ обозначим расстояние от точки $v \in L^{q}(T, Y)$ до множества $B \subset L^{q}(T, Y)$, а через $D_{L}(\cdot, \cdot)$ - обобшенную метрику Хаусдорфа на пространстве $\mathrm{c} L^{q}(T, Y)$, порожденные нормой пространства $L^{q}(T, Y)$.

Пусть $U_{1}, U_{2}: T \rightarrow Y$ - измеримые многозначные отображения с замкнутьми значениями. Через $S_{1}, S_{2}$ обозначим совокупность всех селекторов отображений $U_{1}, U_{2}$, являющихся элементами пространства $L^{q}(T, Y)$. Если множества $S_{1}, S_{2}$ не пусты, то они являются разложимьми, замкнутыми в $L^{q}(T, Y)$ множествами.

УТВЕРЖДЕНИЕ 3.1. Пусть $U_{1}, U_{2}: T \rightarrow \mathrm{cY}$ - измеримые многозначные отображения. Предположим, что множества $S_{1}, S_{2}$ не пусты. Тогда для любого $u \in L^{q}(T, Y)$

$$
\begin{gathered}
d_{L}\left(u, S_{1}\right)=\left(\int_{T} d_{Y}^{q}\left(u(t), U_{1}(t)\right) d t\right)^{1 / q} \\
D_{L}\left(S_{1}, S_{2}\right) \leqslant\left(\int_{T} D_{Y}^{q}\left(U_{1}(t), U_{2}(t)\right) d t\right)^{1 / q} \leqslant 2^{1 / q} D_{L}\left(S_{1}, S_{2}\right) .
\end{gathered}
$$

Доказательство неравенств (3.4), (3.5) проводится по аналогии с доказательством подобных неравенств в [19; утверждение 4.2], где они были доказаны в предположении, что $S_{1}$ и $S_{2}$ являются ограниченньпи подмножествами пространства $L^{q}(T, Y)$.

Рассмотрим уравнение

$$
\begin{gathered}
\dot{x}(t)+A(t, x(t), \xi)=f(t) \quad \text { п.в. } \\
x(0)=\lambda, \quad \xi \in \Lambda .
\end{gathered}
$$

Если выполняются гипотезы $\Gamma(A)$, то в соответствии с $[17 ;$ гл. VI, $\S 1$, теорема 1.1$]$ и $\left[17 ;\right.$ гл. VI, $\S 1$, пример 1] для любых $f \in \mathscr{V}^{*}, \xi \in \Lambda, \lambda \in H$ уравнение (3.6) имеет единственное решение $x(\xi, \lambda, f) \in \mathscr{W} \hookrightarrow C(T, H)$.

Пусть $\lambda \in H$ и $f \in \mathscr{V}^{*}$ фиксированы и $\xi_{n} \rightarrow \xi_{0}, n \geqslant 1$. Тогда будут определены решения $x\left(\xi_{n}, \lambda, f\right), n \geqslant 0$, уравнения (3.6). 
Лемма 3.2. Последовательность $x\left(\xi_{n}, \lambda, f\right), n \geqslant 1$, сходится $к x\left(\xi_{0}, \lambda, f\right)$ в $C(T, H)$.

ДокАЗАТЕЛЬСТво. Вначале рассмотрим случай, когда $f \in C(T, H)$. Пусть $C(T, w-H)$ - пространство всех непрерывных функций из $T$ в $w-H$ с топологией равномерной сходимости на $T$. По аналогии с леммой 3.1 можно показать, что существуют $M_{i}^{*}>0, i=1, \ldots, 4$, такие, что для любых $n \geqslant 0$ справедливы неравенства

$$
\begin{gathered}
\left|x\left(\xi_{n}, \lambda, f\right)(t)\right|_{H} \leqslant M_{1}^{*}, \quad t \in T, \\
\left\|x\left(\xi_{n}, \lambda, f\right)\right\|_{\mathscr{V}} \leqslant M_{2}^{*}, \quad\left\|\dot{x}\left(\xi_{n}, \lambda, f\right)\right\|_{\mathscr{V} *} \leqslant M_{3}^{*}, \\
\left\|x\left(\xi_{n}, \lambda, f\right)\right\|_{\mathscr{W}} \leqslant M_{4}^{*} .
\end{gathered}
$$

Покажем, что для любого $h \in H$ последовательность числовых функций

$$
\left(h, x\left(\xi_{n}, \lambda, f\right)(t)\right), \quad n \geqslant 0,
$$

равностепенно непрерывна. Так как $V$ плотно вложено в $H$, то в соответствии c (3.7) нам достаточно показать, что последовательность (3.10) равностепенно непрерывна для любого $h \in V$. Воспользовавшись формулой интегрирования по частям [17] для элементов из $\mathscr{W}$, мы получим

$$
\begin{aligned}
& \mid(h,\left.x\left(\xi_{n}, \lambda, f\right)(t)-x\left(\xi_{n}, \lambda, f\right)(\tau)\right) \mid \\
& \quad=\left|\int_{\tau}^{t}\left(h, \dot{x}\left(\xi_{n}, \lambda, f\right)(s)\right) d s\right| \leqslant\|h\|_{V}\left\|\dot{x}\left(\xi_{n}, \lambda, f\right)\right\|_{\mathscr{V}^{*}}|t-\tau|^{1 / p} .
\end{aligned}
$$

Из этого неравенства и (3.8) следует, что последовательность (3.10) равностепенно непрерывна. Поскольку любое выпуклое, замкнутое, ограниченное множество из $H$ является метризуемым компактом в $w-H$, то из (3.7) и теоремы Арцела-Асколи следует, что последовательность $x\left(\xi_{n}, \lambda, f\right), n \geqslant 1$, является метризуемьм, относительно компак тньм подмножеством пространства $C(T, w-H)$. Воспользовавшись замечанием 2.2 и теоремой 1 из [11], мы получим, что

$$
x\left(\xi_{n}, \lambda, f\right)(t) \rightarrow x\left(\xi_{0}, \lambda, f\right)(t) \quad \text { в } H, \quad t \in T .
$$

Поэтому

$$
x\left(\xi_{n}, \lambda, f\right) \rightarrow x\left(\xi_{0}, \lambda, f\right) \quad \text { в } C(T, w-H) .
$$

Следовательно, для любой последовательности $t_{n} \rightarrow t_{0}$ мы имеем

$$
x\left(\xi_{n}, \lambda, f\right)\left(t_{n}\right) \rightarrow x\left(\xi_{0}, \lambda, f\right)\left(t_{0}\right) \quad \text { в } w-H .
$$

Из $(3.12)$ и $[17 ;$ гл. I, $\S 5$, лемма 5.3$]$ следует, что

$$
\left|x\left(\xi_{0}, \lambda, f\right)\left(t_{0}\right)\right|_{H} \leqslant \varliminf_{n \rightarrow \infty}\left|x\left(\xi_{n}, \lambda, f\right)\left(t_{n}\right)\right|_{H} .
$$

Предположим, что утверждение леммы неверно. Тогда найдутся $\varepsilon>0$, последовательность $t_{k} \rightarrow t_{0}$ при $k \rightarrow \infty$ и подпоследовательность $x\left(\xi_{n_{k}}, \lambda, f\right), k \geqslant 1$, последовательности $x\left(\xi_{n}, \lambda, f\right), n \geqslant 1$, такие, что

$$
\left|x\left(\xi_{n_{k}}, \lambda, f\right)\left(t_{k}\right)-x\left(\xi_{0}, \lambda, f\right)\left(t_{k}\right)\right|_{H}>\varepsilon, \quad k \geqslant 1 .
$$


Поскольку функция $x\left(\xi_{0}, \lambda, f\right)(t)$ непрерьвна, то

$$
\left|x\left(\xi_{n_{k}}, \lambda, f\right)\left(t_{k}\right)-x\left(\xi_{0}, \lambda, f\right)\left(t_{0}\right)\right|_{H}>\frac{\varepsilon}{2},
$$

если $k \geqslant N$ и $N$ достаточно велико.

Воспользовавшись (3.13) и переходя, если необходимо, к подпоследовательности, мы можем считать, что

$$
\left|x\left(\xi_{0}, \lambda, f\right)\left(t_{0}\right)\right|_{H} \leqslant a=\lim _{k \rightarrow \infty}\left|x\left(\xi_{n_{k}}, \lambda, f\right)\left(t_{k}\right)\right|_{H}
$$

Если $a=\left|x\left(\xi_{0}, \lambda, f\right)\left(t_{0}\right)\right|_{H}$, то из $(3.12),(3.15)$, равномерной выпуклости пространства $H$ и $[17 ;$ гл. I, $\S 5$, теорема 5.12$]$ следует, что

$$
x\left(\xi_{n_{k}}, \lambda, f\right)\left(t_{k}\right) \rightarrow x\left(\xi_{0}, \lambda, f\right)\left(t_{0}\right) \text { в } H
$$

Но это противоречит (3.14). Поэтому в соответствии с (3.15)

$$
a^{2}=\left|x\left(\xi_{0}, \lambda, f\right)\left(t_{0}\right)\right|_{H}^{2}+\varepsilon_{1}
$$

при некотором $\varepsilon_{1}>0$.

Покажем, что при достаточно больших $k$ будет иметь место неравенство $t_{k}<t_{0}$. Если это не так, то, переходя, если это необходимо, к подпоследовательности, мы можем считать, что $t_{0} \leqslant t_{k}$ и $t_{k} \rightarrow t_{0}$ при $k \rightarrow \infty$.

Проинтегрировав уравнение (3.6) с учетом (2.5), мы получим

$$
\begin{aligned}
& \left|x\left(\xi_{n_{k}}, \lambda, f\right)\left(t_{k}\right)\right|_{H}^{2}-\left|x\left(\xi_{n_{k}}, \lambda, f\right)\left(t_{0}\right)\right|_{H}^{2} \\
& \quad \leqslant 2 \int_{t_{0}}^{t_{k}}\left(x\left(\xi_{n_{k}}, \lambda, f\right)(s), f(s)\right) d s \\
& \quad \leqslant 2 \max _{t \in T}\|f(t)\|_{V^{*}}\left\|x\left(\xi_{n_{k}}, \lambda, f\right)\right\|_{\mathcal{V}}\left(t_{k}-t_{0}\right)^{1 / q} .
\end{aligned}
$$

Тогда из $(3.8),(3.11)$ и (3.17) следует, что

$$
a^{2}=\lim _{k \rightarrow \infty}\left|x\left(\xi_{n_{k}}, \lambda, f\right)\left(t_{k}\right)\right|_{H}^{2} \leqslant\left|x\left(\xi_{0}, \lambda, f\right)\left(t_{0}\right)\right|_{H}^{2}
$$

Но это противоречит (3.16). Поэтому $t_{k}<t_{0}$ при достаточно больших $k$ и, следовательно, $t_{0}>0$.

Проинтегрируем уравнение (3.6) по отрезку $\left[t_{k}-\eta, t_{k}\right], \eta=$ const $>0, t_{k}-\eta \geqslant 0$. Учитывая (3.8), по аналогии с (3.17) мы получаем

$$
\left|x\left(\xi_{n_{k}}, \lambda, f\right)\left(t_{k}\right)\right|_{H}^{2} \leqslant c_{1} \eta^{1 / q}+\left|x\left(\xi_{n_{k}}, \lambda, f\right)\left(t_{k}-\eta\right)\right|_{H}^{2}
$$

при некотором $c_{1}>0$. Из этого неравенства и (3.15), (3.16) вытекает, что

$$
\begin{aligned}
\left|x\left(\xi_{n_{k}}, \lambda, f\right)\left(t_{k}-\eta\right)\right|_{H}^{2} & \geqslant\left|x\left(\xi_{n_{k}}, \lambda, f\right)\left(t_{k}\right)\right|_{H}^{2}-\frac{\varepsilon_{1}}{4} \geqslant a^{2}-\frac{\varepsilon_{1}}{2} \\
& \geqslant\left|x\left(\xi_{0}, \lambda, f\right)\left(t_{0}\right)\right|_{H}^{2}+\frac{\varepsilon_{1}}{2}
\end{aligned}
$$


если $0 \leqslant \eta \leqslant \delta_{1}, \delta_{1}=$ const $>0$ достаточно мала, и $k$ достаточно велико. Поскольку функция $x\left(\xi_{0}, \lambda, f\right)(t)$ непрерьвна, то

$$
\left|x\left(\xi_{0}, \lambda, f\right)\left(t_{0}\right)\right|^{2} \geqslant\left|x\left(\xi_{0}, \lambda, f\right)\left(t_{k}-\eta\right)\right|_{H}^{2}-\frac{\varepsilon_{1}}{4},
$$

если $0 \leqslant \eta \leqslant \delta_{2}, t_{0}-t_{k}<\delta_{3}$ и $\delta_{2}, \delta_{3}$ достаточно малы. Поэтому из (3.18) получаем

$$
\left|x\left(\xi_{n_{k}}, \lambda, f\right)\left(t_{k}-\eta\right)\right|_{H}^{2} \geqslant\left|x\left(\xi_{0}, \lambda, f\right)\left(t_{k}-\eta\right)\right|_{H}^{2}+\frac{\varepsilon_{1}}{4}
$$

при $0 \leqslant \eta \leqslant \min \left\{\delta_{1}, \delta_{2}\right\}=\delta_{0}$ и достаточно больших $k$. Так как $t_{k}<t_{0}$, то из определения $\delta_{0}>0$ следует, что сушествует $k_{0}$ такое, что множество $\mathscr{T}=\bigcap_{k} \geqslant k_{0}\left[t_{k}-\delta_{0}, t_{k}\right]$ не пусто. Возьмем произвольное $t \in \mathscr{T}$. Тогда существует $0<\eta_{k}<\delta_{0}$ такое, что $t=t_{k}-\eta_{k}, k \geqslant k_{0}$. Воспользовавшись (3.19), мы получим

$$
\left|x\left(\xi_{n_{k}}, \lambda, f\right)(t)\right|_{H}^{2} \geqslant\left|x\left(\xi_{0}, \lambda, f\right)(t)\right|_{H}^{2}+\frac{\varepsilon_{1}}{4}, \quad k \geqslant k_{0} .
$$

Но последнее неравенство противоречит (3.11). Следовательно, $x\left(\xi_{n_{k}}, \lambda, f\right) \rightarrow$ $x\left(\xi_{0}, \lambda, f\right)$ в $C(T, H)$.

Рассмотрим общий случай. Пусть $u \in \mathscr{V}^{*}$. Так как пространство $C(T, H)$ плотно вложено в $\mathscr{V}^{*}$, то для любого $\varepsilon>0$ найдется $f \in C(T, H)$ такое, что

$$
\|u-f\|_{\mathscr{Y} *}<\varepsilon
$$

Пусть $x\left(\xi_{n}, \lambda, u\right), n \geqslant 0,-$ последовательность решений уравнения (3.6). Как и для последовательности $x\left(\xi_{n}, \lambda, f\right), n \geqslant 0$, можно показать, что для последовательности $x\left(\xi_{n}, \lambda, u\right), n \geqslant 0$, имеют место неравенства, аналогичные (3.7)-(3.9). Тогда сушествует $M>0$ такое, что

$$
\left\|x\left(\xi_{n}, \lambda, f\right)-x\left(\xi_{n}, \lambda, u\right)\right\|_{\mathscr{V}} \leqslant M, \quad n \geqslant 1 .
$$

Воспользовавшись уравнением (3.6), (2.2) и формулой интегрирования по частям для элементов из $\mathscr{W}$, мы получим

$$
\left|x\left(\xi_{n}, \lambda, f\right)(t)-x\left(\xi_{n}, \lambda, u\right)(t)\right|_{H}^{2} \leqslant 2\left\|x\left(\xi_{n}, \lambda, f\right)-x\left(\xi_{n}, \lambda, u\right)\right\|_{\mathscr{V}}\|u-f\|_{\mathscr{V}^{*}},
$$

$n \geqslant 1, t \in T$. Поэтому в соответствии с (3.20), (3.21)

$$
\left|x\left(\xi_{n}, \lambda, f\right)(t)-x\left(\xi_{n}, \lambda, u\right)(t)\right|_{H}^{2}<2 M \varepsilon, \quad n \geqslant 1, \quad t \in T .
$$

Из (3.22) следует, что

$$
\bigcup_{n \geqslant 1} x\left(\xi_{n}, \lambda, u\right) \subset \bigcup_{n \geqslant 0} x\left(\xi_{n}, \lambda, f\right)+(2 M \varepsilon)^{1 / 2} B_{C(T, H)},
$$

где $B_{C(T, H)}$ - единичньй открытый шар из $C(T, H)$. Поскольку множество $\bigcup_{n \geqslant 0} x\left(\xi_{n}, \lambda, f\right)$, как было доказано выше, является компактом в $C(T, H)$, то включение (3.23) означает, что для любого $\varepsilon>0$ существует компактное множество $\bigcup_{n \geqslant 0} x\left(\xi_{n}, \lambda, f\right)$ такое, что имеет место включение (3.23). Поэтому 
множество $\bigcup_{n \geqslant 1} x\left(\xi_{n}, \lambda, u\right)$ относительно компактно в $C(T, H)$. Согласно [11; теорема 1]

$$
x\left(\xi_{n}, \lambda, u\right)(t) \rightarrow x\left(\xi_{0}, \lambda, u\right)(t) \quad \text { в } \quad H, \quad t \in T .
$$

Следовательно, последовательность $x\left(\xi_{n}, \lambda, u\right), n \geqslant 1$, сходится в $C(T, H)$ к $x\left(\xi_{0}, \lambda, u\right)$. Лемма доказана.

Пусть выполняются гипотезы $\Gamma(B)$. Тогда для любого $u \in L^{q}(T, Y)$ функция $f(t)=B(t) u(t), t \in T$, является элементом пространства $\mathscr{H}^{*}$. Поэтому на основании вышесказанного для любых $\xi \in \Lambda, \lambda \in H, u \in L^{q}(T, Y)$ уравнение (1.1) имеет единственное решение $x(\xi, \lambda, u) \in \mathscr{W} \hookrightarrow C(T, H)$. Тем самым будет определен оператор $\mathscr{T}: \Lambda \times H \times L^{q}(T, Y) \rightarrow \mathscr{W} \hookrightarrow C(T, H)$, который каждой тройке $(\xi, \lambda, u)$ ставит в соответствие единственное решение

$$
x(\xi, \lambda, u)=\mathscr{T}(\xi, \lambda, u)
$$

уравнения (1.1).

Следующая теорема является основополагающей в наших исследованиях.

ТЕОРема 3.1. Пусть выполняются гипотезы $\Gamma(A), \Gamma(B)$. Тогда отображение $(\xi, \lambda, u) \mapsto \mathscr{T}(\xi, \lambda, u)$ является секвенциально непрерывным из $\Lambda \times H \times$ $w-L^{q}(T, Y)$ в $C(T, H)$.

ДОкаЗАТЕЛЬСтво. Пусть $\xi_{n} \rightarrow \xi_{0}$ в $\Lambda, \lambda_{n} \rightarrow \lambda_{0}$ в $H$ и $u_{n} \rightarrow u_{0}$ в $w-L^{q}(T, Y)$. Положим

$$
\begin{aligned}
y_{n} & =\mathscr{T}\left(\xi_{n}, \lambda_{n}, u_{n}\right), \quad n \geqslant 1, \\
x_{n} & =\mathscr{T}\left(\xi_{n}, \lambda_{0}, u_{0}\right), \quad n \geqslant 1, \\
x_{0} & =\mathscr{T}\left(\xi_{0}, \lambda_{0}, u_{0}\right) .
\end{aligned}
$$

По аналогии с леммой 3.1 можно показать, что для последовательностей $y_{n}, x_{n}$, $n \geqslant 1$, имеют место оценки (3.7)-(3.9). Рассмотрим последовательность $z_{n}=$ $y_{n}-x_{n}, n \geqslant 1$. Воспользовавшись неравенством (3.9), мы получим, что последовательность $z_{n}, n \geqslant 1$, относительно компактна в $w$ - $\mathscr{W}$ и, следовательно, является относительно компактным множеством в $\mathscr{H}$. Пусть $v_{n}(t)=B(t) u_{n}(t), n \geqslant 0$. Из гипотез Г $(B)$ следует, что

$$
v_{n} \rightarrow v_{0} \quad \text { в } w-\mathscr{H}^{*} .
$$

Воспользовавшись уравнением (1.1) и (2.2), мы получим

$$
\frac{1}{2}\left|y_{n}(t)-x_{n}(t)\right|_{H}^{2} \leqslant \frac{1}{2}\left|\lambda_{n}-\lambda_{0}\right|_{H}^{2}+\int_{0}^{t}\left(y_{n}(s)-x_{n}(s), v_{n}(s)-v_{0}(s)\right) d s .
$$

Зафиксируем $t \in T$ и перепишем последнее неравенство в виде

$$
\frac{1}{2}\left|z_{n}(t)\right|_{H}^{2} \leqslant \frac{1}{2}\left|\lambda_{n}-\lambda_{0}\right|_{H}^{2}+\int_{T}\left(z_{n}(s), f_{n}(s)-f_{0}(s)\right) d s,
$$

где $f_{n}(s)=\chi_{t}(s) v_{n}(s), s \in T, n \geqslant 0$ и $\chi_{t}(s)$ - характеристическая функция отрезка $[0, t]$. Из (3.28) следует, что

$$
f_{n} \rightarrow f_{0} \quad \text { в } w-\mathscr{H}^{*} .
$$


Пусть $\left(y, y^{\prime}\right) \mathscr{H}$ - каноническая билинейная форма, устанавливающая двойственность между $\mathscr{H}$ и $\mathscr{H}^{*}$. Из (3.31) следует, что последовательность числовых функций $y \mapsto\left(y, f_{n}\right) \mathscr{H}, n \geqslant 1, y \in \mathscr{H}$, равностепенно непрерывна [16]. Пусть $D=$ $\left\{\bigcup_{n \geqslant 1} z_{n}\right\} \subset \mathscr{H}$. Известно, что на каждом равностепенно непрерывном множестве топология поточечной сходимости совпадает с топологией равномерной сходимости на компактах из $\mathscr{H}$. Поскольку множество $D$ относительно компактно в $\mathscr{H}$, то в соответствии с (3.31)

$$
\lim _{n \rightarrow \infty} \sup _{y \in D}\left|\left(y, f_{n}-f_{0}\right) \mathscr{H}\right|=\lim _{n \rightarrow \infty} \sup _{y \in D}\left|\int_{T}\left(y(s), f_{n}(s)-f_{0}(s)\right) d s\right|=0
$$

Теперь из (3.30), (3.32) следует, что

$$
\left|z_{n}(t)\right|_{H}=\left|y_{n}(t)-x_{n}(t)\right|_{H} \rightarrow 0, \quad t \in T
$$

Так как последовательность $z_{n}, n \geqslant 1$, относительно компактна в $\mathscr{H}$, то в соответствии с (3.33)

$$
z_{n} \rightarrow \Theta_{\mathscr{H}} \quad \text { в } \mathscr{H}
$$

где $\Theta \mathscr{H}$ - нулевой элемент пространства $\mathscr{H}$. Из (3.29) следует, что

$$
\frac{1}{2}\left|y_{n}(t)-x_{n}(t)\right|_{H}^{2} \leqslant \frac{1}{2}\left|\lambda_{n}-\lambda_{0}\right|_{H}^{2}+\left\|z_{n}\right\| \mathscr{H}\left\|v_{n}-v_{0}\right\|_{\mathscr{H}^{*}}
$$

Из последнего неравенства, (3.28), (3.34) следует, что

$$
\lim _{n \rightarrow \infty} \sup _{t \in T}\left|y_{n}(t)-x_{n}(t)\right|_{H}=0
$$

Воспользовавшись (3.25)-(3.27), (3.35), леммой 3.2 и неравенством

$$
\left|y_{n}(t)-x_{0}(t)\right|_{H} \leqslant\left|y_{n}(t)-x_{n}(t)\right|_{H}+\left|x_{n}(t)-x_{0}(t)\right|_{H},
$$

мы получим, что

$$
\mathscr{T}\left(\xi_{n}, \lambda_{n}, u_{n}\right) \rightarrow \mathscr{T}\left(\xi_{0}, \lambda_{0}, u_{0}\right) \text { в } C(T, H)
$$

Теорема доказана.

ЗАМЕчАНИЕ 3.1. При доказательстве леммы 3.2 мы использовали только условия, при которых уравнение (3.6) имеет единственное решение и справедливо утверждение основной теоремы 1 из [11]. Все это имеет место и в рамках предположений А)-D) этой теоремы. Поэтому лемма 3.2 является усилением теоремы 1 из [11], где утверждается только поточечная сходимость последовательности $x\left(\xi_{n}, \lambda, f\right)$, $n \geqslant 1, \mathrm{\text {к }} x\left(\xi_{0}, \lambda, f\right)$. 


\section{§4. Многозначный оператор Немыцкого}

Всюду в этом параграфе, если не оговорено противное, мы предполагаем, что имеют место гипотезы $\Gamma(B), \Gamma(U) 1)-4)$.

Для каждых $(\xi, \lambda, u) \in \Lambda \times H \times L^{q}(T, Y), 1 / p+1 / q=1, p \geqslant 2$, положим

$$
\Phi(\xi, \lambda, u)=\left\{\varphi \in L^{q}(T, Y): \varphi(t) \in U(t, \mathscr{T}(\xi, \lambda, u)(t), \xi) \text { п.в. }\right\},
$$

где $\mathscr{T}(\xi, \lambda, u)$ - оператор (3.24). В соответствии с гипотезами $\Gamma(U) 1), 3)$ для любой непрерывной функции $x: T \rightarrow H$ отображение $t \mapsto U(t, x(t), \xi)$ измеримо. Воспользовавшись неравенством (2.4), мы получим

$$
d_{Y}\left(\Theta_{Y}, U(t, x(t), \xi)\right) \leqslant d_{Y}\left(\Theta_{Y}, U\left(t, \Theta_{H}, \xi\right)\right)+l(\xi)(t)|x(t)|_{H} .
$$

Из этого неравенства, гипотезы $\Gamma(U) 4)$ и свойств измеримых многозначных отображений $[20]$ вытекает, что для каждых $(\xi, \lambda, u)$ множество $\Phi(\xi, \lambda, u)$ является непустым, разложимьм, замкнутым подмножеством пространства $L^{q}(T, Y)$. Поэтому будет определено отображение $\Phi: \Lambda \times H \times L^{q}(T, Y) \rightarrow \mathrm{c} L^{q}(T, Y)$, которое называется многозначным оператором Немьцкого.

Наряду с оператором $\Phi$ рассмотрим оператор $\overline{\operatorname{co}} \Phi,(\overline{c o} \Phi)(\xi, \lambda, u)=\overline{\operatorname{co}} \Phi(\xi, \lambda, u)$. Известно [20], что

$$
\overline{\mathrm{CO}} \Phi(\xi, \lambda, u)=\left\{\varphi \in L^{q}(T, Y): \varphi(t) \in \overline{\mathrm{co}} U(t, \mathscr{T}(\xi, \lambda, u)(t), \xi) \text { п.в. }\right\} .
$$

На пространстве $\Lambda \times L^{q}(T, Y)$ рассмотрим числовую функцию

$$
P(\xi, u)=\left(\int_{T} \rho^{q}(t, \xi, u(t)) d t\right)^{1 / q}
$$

где

$$
\rho(t, \xi, u)=\exp \left(-\frac{1}{q} m^{q} 2^{q} \int_{0}^{t} l^{q}(\xi)(\tau) d \tau\right)\|u\|_{Y}
$$

где $m$ - константа, фигурируюшая в неравенстве $(2.3)$, а $l(\xi)(\tau)$ - функция из неравенства (2.4). Тогда функция $\rho(t, \xi, u)$ обладает свойствами $1 \rho)-3 \rho)$, введенными в $§ 2$ работы [12], а функция $P(\xi, u)$ удовлетворяет неравенству, аналогичному неравенству (3.1) в [12]. Поэтому для каждого $\xi \in \Lambda$ функция $P(\xi, \cdot)$ является нормой, эквивалентной исходной норме пространства $L^{q}(T, Y)$.

Для каждого $\xi \in \Lambda$ через $d_{L}(\xi)(u, B)$ и $D_{L}(\xi)(\cdot, \cdot)$ обозначим расстояние от точки $u \in L^{q}(T, Y)$ до множества $B \subset L^{q}(T, Y)$ и обобщенную метрику Хаусдорфа, порожденные нормой $P(\xi, \cdot)$.

TЕОРема 4.1. Пусть выполняются гипотезь $\Gamma(U)$ 1)-4). Тогда оператор Немьцкого $\Phi: \Lambda \times H \times L^{q}(T, Y) \rightarrow \mathrm{c} L^{q}(T, Y)$ обладает следующими свойствами:

1) отображение $(\xi, \lambda) \mapsto \Phi(\xi, \lambda, u)$ полунепрерывно снизу по Вветорису;

2) справедливо

$$
D_{L}(\xi)(\Phi(\xi, \lambda, u), \Phi(\xi, \lambda, v)) \leqslant \frac{1}{2} P(\xi, u-v),
$$

$$
\xi \in \Lambda, \lambda \in H, u, v \in L^{q}(T, Y)
$$


3) справедливо

$$
\begin{aligned}
& D_{L}\left(\Phi\left(\xi, \lambda_{1}, u_{1}\right), \Phi\left(\xi, \lambda_{2}, u_{2}\right)\right) \\
& \quad \leqslant\|l(\xi)\|_{L^{q}\left(T, \mathbb{R}^{+}\right)}\left(\left|\lambda_{1}-\lambda_{2}\right|_{H}+m\left\|u_{1}-u_{2}\right\|_{L^{q}(T, Y)}\right),
\end{aligned}
$$

$\xi \in \Lambda, \lambda_{1}, \lambda_{2} \in H, u_{1}, u_{2} \in L^{q}(T, Y)$

4) отображение $(\xi, \lambda, u) \mapsto \Phi(\xi, \lambda, u)$ имеет замкнутый график в $\Lambda \times H \times$ $L^{q}(T, Y) \times L^{q}(T, Y)$.

ДоказАТЕЛЬство. Покажем вначале, что отображение $\xi \mapsto \Phi(\xi, \lambda, u)$ полунепрерывно снизу по Вьеторису для фиксированных $\lambda \in H, u \in L^{q}(T, Y)$.

Пусть последовательность $\xi_{n} \in \Lambda, n \geqslant 1$, сходится к $\xi$. Возьмем произвольное $\varphi \in \Phi(\xi, \lambda, u)$. В соответствии с гипотезой $\Gamma(U) 2)$ отображение $\tau \mapsto$ $U(t, \mathscr{T}(\xi, \lambda, u)(t), \tau)$ полунепрерьвно снизу по Вьеторису. Поэтому

$$
\lim _{n \rightarrow \infty} d_{Y}\left(\varphi(t), U\left(t, \mathscr{T}(\xi, \lambda, u)(t), \xi_{n}\right)\right)=0 \text { п.в. }
$$

Так как

$$
\begin{aligned}
& d_{Y}\left(\varphi(t), U\left(t, \mathscr{T}(\xi, \lambda, u)(t), \xi_{n}\right)\right) \\
& \quad \leqslant d_{Y}\left(\varphi(t), U\left(t, \Theta_{H}, \xi_{n}\right)\right)+D_{Y}\left(U\left(t, \Theta_{H}, \xi_{n}\right), U\left(t, \mathscr{T}(\xi, \lambda, u)(t), \xi_{n}\right)\right) \\
& \quad \leqslant\|\varphi(t)\|_{Y}+d_{Y}\left(\Theta_{Y}, U\left(t, \Theta_{H}, \xi_{n}\right)\right)+l\left(\xi_{n}\right)(t)\|\mathscr{T}(\xi, \lambda, u)\|_{C(T, H)},
\end{aligned}
$$

то из гипотезы $\Gamma(U) 4)$ и последнего неравенства получаем, что последовательность $d_{Y}\left(\varphi(t), U\left(t, \mathscr{T}(\xi, \lambda, u)(t), \xi_{n}\right)\right), n \geqslant 1$, равномерно $q$-интегрируема. Поэтому из (4.7) и $[19 ;$ утверждение 2.4$]$ мы получаем, что

$$
\lim _{n \rightarrow \infty}\left(\int_{T} d_{Y}^{q}\left(\varphi(t), U\left(t, \mathscr{T}(\xi, \lambda, u)(t), \xi_{n}\right)\right) d t\right)^{1 / q}=0 .
$$

Поскольку

$$
\begin{aligned}
& d_{Y}\left(\varphi(t), U\left(t, \mathscr{T}\left(\xi_{n}, \lambda, u\right)(t), \xi_{n}\right)\right) \\
& \quad \leqslant d_{Y}\left(\varphi(t), U\left(t, \mathscr{T}(\xi, \lambda, u)(t), \xi_{n}\right)\right)+l\left(\xi_{n}\right)(t)\left\|\mathscr{T}\left(\xi_{n}, \lambda, u\right)-\mathscr{T}(\xi, \lambda, u)\right\|_{C(T, H)},
\end{aligned}
$$

то из (4.8), (3.4) и теоремы 3.1 мы получаем, что

$$
\lim _{n \rightarrow \infty} d_{L}\left(\varphi, \Phi\left(\xi_{n}, \lambda, u\right)\right)=0 .
$$

Но это означает, что отображение $\xi \mapsto \Phi(\xi, \lambda, u)$ полунепрерывно снизу по Вьеторису.

В дальнейшем нам понадобится следуюшее неравенство. Пусть

$$
x\left(\xi, \lambda_{1}, u_{1}\right)=\mathscr{T}\left(\xi, \lambda_{1}, u_{1}\right), \quad x\left(\xi, \lambda_{2}, u_{2}\right)=\mathscr{T}\left(\xi, \lambda_{2}, u_{2}\right) .
$$

Тогда

$\left|x\left(\xi, \lambda_{1}, u_{1}\right)(t)-x\left(\xi, \lambda_{2}, u_{2}\right)(t)\right|_{H} \leqslant\left|\lambda_{1}-\lambda_{2}\right|_{H}+\int_{0}^{t} m\left\|u_{1}(s)-u_{2}(s)\right\|_{Y} d s, \quad t \in T$. 
Воспользовавшись уравнением (1.1) и (2.3), мы получим

$$
\begin{aligned}
& \frac{1}{2}\left|x\left(\xi, \lambda_{1}, u_{1}\right)(t)-x\left(\xi, \lambda_{2}, u_{2}\right)(t)\right|_{H}^{2} \\
& \quad \leqslant \frac{1}{2}\left|\lambda_{1}-\lambda_{2}\right|_{H}^{2}+\int_{0}^{t}\left(x\left(\xi, \lambda_{1}, u_{1}\right)(s)-x\left(\xi, \lambda_{2}, u_{2}\right)(s), B(s) u_{1}(s)-B(s) u_{2}(s)\right) d s \\
& \quad \leqslant \frac{1}{2}\left|\lambda_{1}-\lambda_{2}\right|_{H}^{2}+\int_{0}^{t} m\left|x\left(\xi, \lambda_{1}, u_{1}\right)(s)-x\left(\xi, \lambda_{2}, u_{2}\right)(s)\right|_{H}\left\|u_{1}(s)-u_{2}(s)\right\|_{Y} d s .
\end{aligned}
$$

Теперь неравенство (4.9) вытекает из (4.10) и [21; лемма А5].

Покажем теперь, что отображение $(\xi, \lambda) \mapsto \Phi(\xi, \lambda, u)$ полунепрерывно снизу по Вьеторису для каждого $u \in L^{q}(T, Y)$. Пусть последовательность $\left(\xi_{n}, \lambda_{n}\right), n \geqslant 1$, сходится к $(\xi, \lambda)$. Возьмем произвольное $\varphi \in \Phi(\xi, \lambda, u)$. Тогда из $(2.4)$ и $(4.9)$ мы получим

$$
\begin{aligned}
& d_{Y}\left(\varphi(t), U\left(t, \mathscr{T}\left(\xi_{n}, \lambda_{n}, u\right)(t), \xi_{n}\right)\right) \\
& \quad \leqslant d_{Y}\left(\varphi(t), U\left(t, \mathscr{T}\left(\xi_{n}, \lambda, u\right)(t), \xi_{n}\right)\right)+l\left(\xi_{n}\right)(t)\left|\lambda_{n}-\lambda\right|_{H} .
\end{aligned}
$$

Воспользовавшись (3.4), (4.1), (4.11), мы придем к неравенству

$$
d_{L}\left(\varphi, \Phi\left(\xi_{n}, \lambda_{n}, u\right)\right) \leqslant d_{L}\left(\varphi, \Phi\left(\xi_{n}, \lambda, u\right)\right)+\left|\lambda_{n}-\lambda\right|_{H}\left(\int_{T} l^{q}\left(\xi_{n}\right)(t) d t\right)^{1 / q} .
$$

Так как отображение $\xi \mapsto \Phi(\xi, \lambda, u)$ полунепрерывно снизу по Вьеторису, то из (4.12) следует, что

$$
\lim _{n \rightarrow \infty} d_{L}\left(\varphi, \Phi\left(\xi_{n}, \lambda_{n}, u\right)\right)=0 .
$$

Но это означает, что отображение $(\xi, \lambda) \mapsto \Phi(\xi, \lambda, u)$ полунепрерьвно снизу по Вьеторису. Тем самьм утверждение 1) теоремы доказано.

Докажем неравенство (4.5). Из (2.4), (4.9) следует, что

$$
D_{Y}(U(t, \mathscr{T}(\xi, \lambda, u)(t), \xi), U(t, \mathscr{T}(\xi, \lambda, v)(t), \xi)) \leqslant m l(\xi)(t) \int_{0}^{t}\|u(s)-v(s)\|_{Y} d s
$$

Тогда, воспользовавшись (4.13), (4.1), (4.3), (4.4) и первым неравенством в (3.5), мы получим

$$
\begin{aligned}
& D_{L}(\xi)(\Phi(\xi, \lambda, u), \Phi(\xi, \lambda, v)) \\
& \quad \leqslant\left(\int_{T} \exp \left(-m^{q} 2^{q} \int_{0}^{t} l^{q}(\xi)(\tau) d \tau\right) m^{q} l^{q}(\xi)(t)\left(\int_{0}^{t}\|u(s)-v(s)\|_{Y}^{q} d s\right) d t\right)_{(4.14)}^{1 / q} .
\end{aligned}
$$

Проинтегрировав по частям правую часть (4.14), мы получим

$$
\begin{aligned}
& D_{L}(\xi)(\Phi(\xi, \lambda, u), \Phi(\xi, \lambda, v)) \\
& \quad \leqslant \frac{1}{2}\left(\int_{T} \exp \left(-\frac{1}{q} m^{q} 2^{q} \int_{0}^{t} l^{q}(\xi)(\tau) d \tau\right)\|u(t)-v(t)\|_{Y}^{q} d t\right)^{1 / q}
\end{aligned}
$$


Теперь неравенство (4.5) вытекает из (4.3), (4.4) и (4.15).

Докажем неравенство (4.6). Из (2.4) и (3.24) следует, что

$$
\begin{gathered}
D_{Y}\left(U\left(t, \mathscr{T}\left(\xi, \lambda_{1}, u_{1}\right)(t), \xi\right), U\left(t, \mathscr{T}\left(\xi, \lambda_{2}, u_{2}\right)(t), \xi\right)\right) \\
\leqslant l(\xi)(t)\left\|\mathscr{T}\left(\xi, \lambda_{1}, u_{1}\right)-\mathscr{T}\left(\xi, \lambda_{2}, u_{2}\right)\right\|_{C(T, H)}
\end{gathered}
$$

Воспользовавшись (4.16), (4.9), (4.1) и первым из неравенств (3.5), мы придем к неравенству (4.6).

Перейдем к доказательству утверждения 4). Пусть последовательность $\left(\xi_{n}, \lambda_{n}, u_{n}\right), n \geqslant 1$, сходится к $(\xi, \lambda, u)$, а последовательность $\varphi_{n} \in \Phi\left(\xi_{n}, \lambda_{n}, u_{n}\right)$, $n \geqslant 1$, сходится к $\varphi$. Нам нужно доказать, что $\varphi \in \Phi(\xi, \lambda, u)$. Так как $\varphi_{n}(t) \in$ $U\left(t, \mathscr{T}\left(\xi_{n}, \lambda_{n}, u_{n}\right)(t), \xi_{n}\right)$ п.в., то сушествует последовательность измеримых функций $q_{n}: T \rightarrow Y$ такая, что

$$
\begin{gathered}
q_{n}(t) \in U\left(t, \mathscr{T}(\xi, \lambda, u)(t), \xi_{n}\right) \text { п.в., } \\
\left\|\varphi_{n}(t)-q_{n}(t)\right\|_{Y} \leqslant \frac{1}{n}+d_{Y}\left(\varphi_{n}(t), U\left(t, \mathscr{T}(\xi, \lambda, u)(t), \xi_{n}\right)\right) .
\end{gathered}
$$

Воспользовавшись (4.18), (2.4) и (3.24), мы получим

$$
\left\|\varphi(t)-q_{n}(t)\right\|_{Y} \leqslant \frac{1}{n}+\left\|\varphi(t)-\varphi_{n}(t)\right\|_{Y}+l\left(\xi_{n}\right)(t)\left\|\mathscr{T}\left(\xi_{n}, \lambda_{n}, u_{n}\right)-\mathscr{T}(\xi, \lambda, u)\right\|_{C(T, H)}
$$

Поэтому из (4.19) и теоремњ 3.1 вытекает, что $q_{n} \rightarrow \varphi$ в $L^{q}(T, Y)$. Не ограничивая обшности, мы можем считать, что $q_{n}(t) \rightarrow \varphi(t)$ п.в. в $Y$. Тогда из $(4.17)$ и гипотезы $\Gamma(U) 2)$ следует, что $\varphi(t) \in U(t, \mathscr{T}(\xi, \lambda, u)(t), \xi)$ п.в. Следовательно, $\varphi \in \Phi(\xi, \lambda, u)$. Тем самым показано, что отображение $(\xi, \lambda, u) \mapsto \Phi(\xi, \lambda, u)$ имеет замкнутьй график в $\Lambda \times H \times L^{q}(T, Y) \times L^{q}(T, Y)$. Теорема доказана.

Лемма 4.1. Пусть выполняются гипотезы $Г(U) 1)-3)$ и неравенство (2.6). Тогда отображсене $(\xi, \lambda, u) \mapsto \Phi(\xi, \lambda, u)$ является секвенииально непрерывнылм из $\Lambda \times H \times w-L^{q}(T, Y)$ в $\left(\mathrm{cb} L^{q}(T, Y), D_{L}(\cdot, \cdot)\right)$ и справедливы все утверждения теоремы 4.1 .

ДокАЗАТЕльство. Из неравенства (2.6) вытекает, что значениями оператора $\Phi(\xi, \lambda, u)$ являются элементы пространства $\operatorname{cb} L^{q}(T, Y)$. Пусть $\left(\xi_{n}, \lambda_{n}, u_{n}\right) \rightarrow$ $(\xi, \lambda, u)$ в $\Lambda \times H \times w-L^{q}(T, Y)$. Тогда

$$
\begin{aligned}
D_{Y}( & \left.U\left(t, \mathscr{T}\left(\xi_{n}, \lambda_{n}, u_{n}\right)(t), \xi_{n}\right), U(t, \mathscr{T}(\xi, \lambda, u)(t), \xi)\right) \\
\leqslant & l\left(\xi_{n}\right)(t)\left\|\mathscr{T}\left(\xi_{n}, \lambda_{n}, u_{n}\right)-\mathscr{T}(\xi, \lambda, u)\right\|_{C(T, H)} \\
& +D_{Y}\left(U\left(t, \mathscr{T}(\xi, \lambda, u)(t), \xi_{n}\right), U(t, \mathscr{T}(\xi, \lambda, u)(t), \xi)\right) .
\end{aligned}
$$

Из этого неравенства и (3.5) следует, что

$$
\begin{aligned}
D_{L}( & \left.\Phi\left(\xi_{n}, \lambda_{n}, u_{n}\right), \Phi(\xi, \lambda, u)\right) \\
\leqslant & \left\|l\left(\xi_{n}\right)\right\|_{L^{q}\left(T, \mathbb{R}^{+}\right)}\left\|\mathscr{T}\left(\xi_{n}, \lambda_{n}, u_{n}\right)-\mathscr{T}(\xi, \lambda, u)\right\|_{C(T, H)} \\
\quad & +\left(\int_{T} D_{Y}^{q}\left(U\left(t, \mathscr{T}(\xi, \lambda, u)(t), \xi_{n}\right), U(t, \mathscr{T}(\xi, \lambda, u)(t), \xi)\right) d t\right)^{1 / q}
\end{aligned}
$$


Из этого неравенства, теоремы 3.1, гипотезы $\Gamma(U) 2)$, неравенства $(2.6)$ и теоремы Лебега об ограниченной сходимости вытекает, что

$$
\lim _{n \rightarrow \infty} D_{L}\left(\Phi\left(\xi_{n}, \lambda_{n}, u_{n}\right), \Phi(\xi, \lambda, u)\right)=0 .
$$

Следовательно, отображение $(\xi, \lambda, u) \mapsto \Phi(\xi, \lambda, u)$ является секвенциально непрерывным из $\Lambda \times H \times w-L^{q}(T, Y)$ в $\left(\operatorname{cb} L^{q}(T, Y), D_{L}(\cdot, \cdot)\right)$. Отсюда сразу вытекают утверждения 1) и 4) теоремы 4.1. Что же касается неравенств (4.5) и (4.6), то их доказательство базировалось на гипотезах $\Gamma(U) 3), 4)$. Поскольку гипотеза $\Gamma(U) 4)$ следует из неравенства (2.6), то лемма доказана.

\section{§5. Свойства неподвижных точек оператора Немыцкого}

В этом параграфе мы изучим свойства неподвижных точек оператора $\Phi(\xi, \lambda, u)$. Наряду со стандартной нормой на пространстве $L^{q}(T, Y)$ мы будем рассматривать так называемую слабую норму [22]

$$
\|u\|_{\omega}=\sup _{0 \leqslant t \leqslant t^{\prime} \leqslant 1}\left\|\int_{t}^{t^{\prime}} u(s) d s\right\|_{Y} .
$$

Пространство $L^{q}(T, Y)$ с нормой $(5.1)$ мы будем обозначать через $L_{w}^{q}(T, Y)$. Для фиксированных $(\xi, \lambda)$ обозначим через $\operatorname{Fix} \Phi(\xi, \lambda)$ множество неподвижных точек оператора $\Phi(\xi, \lambda, u)$.

ТЕОРема 5.1. Пусть въполняются гипотезъ $\Gamma(U)$ 1)-4). Тогда

а) для любих $(\xi, \lambda)$ множество $\operatorname{Fix} \Phi(\xi, \lambda)$ не пусто и существует непрерввная функиия $u: \Lambda \times H \rightarrow L^{q}(T, Y)$ такая, что

$$
u(\xi, \lambda) \in \operatorname{Fix} \Phi(\xi, \lambda), \quad(\xi, \lambda) \in \Lambda \times H ;
$$

b) если $D \subset \Lambda \times H-$ замкнутое множество и $u_{D}: D \rightarrow L^{q}(T, Y)$ - непрерьвная функиия, $u_{D}(\xi, \lambda) \in \operatorname{Fix} \Phi(\xi, \lambda),(\xi, \lambda) \in D$, то существует непрерьвная функиия $u: \Lambda \times H \rightarrow L^{q}(T, Y)$ такая, что справедливо включение $(5.2)$ и $и(\xi, \lambda)=u_{D}(\xi, \lambda),(\xi, \lambda) \in D$.

СлЕДСТВИЕ 5.1. Пусть выполняются предположения теоремы 5.1. Тогда

а) отобрахсение $(\xi, \lambda) \mapsto \operatorname{Fix} \Phi(\xi, \lambda, u)$ является полунепрерывным снизу по Вветорису с замкнутыми в $L^{q}(T, Y)$ значениями;

b) для любых $(\xi, \lambda)$ множество $\operatorname{Fix} \Phi(\xi, \lambda)$ является абсолютным ретрактом, и ретракцию можнн выбрать непрерывно зависящей от $(\xi, \lambda)$, т.е. существует непрерывная функция $g: \Lambda \times H \times L^{q}(T, Y) \rightarrow$ $L^{q}(T, Y)$ такая, что

$$
\begin{gathered}
g(\xi, \lambda, u) \in \operatorname{Fix} \Phi(\xi, \lambda), \quad(\xi, \lambda) \in \Lambda \times H, \quad u \in L^{q}(T, Y), \\
g(\xi, \lambda, u)=u, \quad u \in \operatorname{Fix} \Phi(\xi, \lambda), \quad(\xi, \lambda) \in \Lambda \times H
\end{gathered}
$$

с) если пространство $\Lambda$ дугообразно связно, то множество

$$
\left\{\bigcup_{(\xi, \lambda) \in \Lambda \times H} \operatorname{Fix} \Phi(\xi, \lambda)\right\}
$$

дугообразно связно; 
d) для любых непрерывных селекторов $u_{i}: \Lambda \times H \rightarrow L^{q}(T, Y), i=1,2$, отобрахсения $\operatorname{Fix} \Phi(\xi, \lambda)$ существует непрерывное отображсние $h: \Lambda \times$ $H \times[0,1] \rightarrow L^{q}(T, Y)$ maкое, что

$$
\begin{gathered}
h(\xi, \lambda, 0)=u_{1}(\xi, \lambda), \quad h(\xi, \lambda, 1)=u_{2}(\xi, \lambda), \quad(\xi, \lambda) \in \Lambda \times H, \\
h(\xi, \lambda, \tau) \in \operatorname{Fix} \Phi(\xi, \lambda), \quad(\xi, \lambda, \tau) \in \Lambda \times H \times[0,1] .
\end{gathered}
$$

Если пространство $\Lambda$ дугообразно связно, то и пространство $\Lambda \times H$ является дугообразно связным. При выполнении гипотез $\Gamma(U) 1)-4)$ справедливы утверждения теоремы 4.1. Эти утверждения являются предположениями в теоремах $3.1,4.1$ и следствии 4.1 из работы [12]. Поэтому теорема 5.1 вытекает из теоремы 3.1 в [12], а утверждения следствия 5.1 являются перефразировкой аналогичных утверждений из теоремы 4.1 и следствия 4.1 в [12].

ЛЕМма 5.1. Пусть выполняются гипотезы $Г(U)$ 1)-3) и неравенство (2.6). Тогда для отображений $\operatorname{Fix} \Phi(\xi, \lambda)$ u $\operatorname{Fix} \overline{c 0} \Phi(\xi, \lambda)$ справедливь все утверждения теоремы 5.1 и следствия 5.1. Более того, отображсение $(\xi, \lambda) \mapsto$ $\operatorname{Fix} \overline{\operatorname{co}} \Phi(\xi, \lambda)$ является непрерывным по Вветорису из $\Lambda \times H$ в $w-L^{q}(T, Y)$ с компактными в $w-L^{q}(T, Y)$ значениями.

ДокАЗАТЕЛЬСтво. В соответствии с замечанием 2.3 из гипотез $\Gamma(U) 1)-3)$ и неравенства (2.6) следует, что для отображения $\overline{\mathrm{co}} U(t, x, \xi)$ имеют место гипотезы $\Gamma(U) 1)-4)$. Воспользовавшись (4.2), мы получим, что для отображений $\mathrm{Fix} \Phi(\xi, \lambda)$ и $\operatorname{Fix} \overline{c o} \Phi(\xi, \lambda)$ справедливы утверждения теоремы 5.1 и следствия 5.1.

Докажем непрерывность по Вьеторису отображения $(\xi, \lambda) \mapsto \operatorname{Fix} \overline{\operatorname{co}} \Phi(\xi, \lambda)$ из $\Lambda \times H$ в $w-L^{q}(T, Y)$.

Поскольку любое открытое в $w-L^{q}(T, Y)$ множество является открытым и в $L^{q}(T, Y)$, то полунепрерывность снизу по Вьеторису отображения $\operatorname{Fix} \overline{c o} \Phi(\xi, \lambda)$ из $\Lambda \times H$ в $w-L^{q}(T, Y)$ вытекает из утверждения а) следствия 5.1. Чтобы доказать полунепрерывность сверху по Вьеторису отображения $\mathrm{Fix} \overline{\mathrm{co}} \Phi(\xi, \lambda)$ из $\Lambda \times H$ в $w-L^{q}(T, Y)$, достаточно показать, что этим свойством обладает сужение отображения $\operatorname{Fix} \overline{\operatorname{co}} \Phi(\xi, \lambda)$ на любое компактное множество $Q \subset \Lambda \times H$.

Пусть $Q \subset \Lambda \times H-$ компактное множество и $u(\xi, \lambda) \in \operatorname{Fix} \overline{c o} \Phi(\xi, \lambda)$. Положим $x(\xi, \lambda)=\mathscr{T}(\xi, \lambda, u(\xi, \lambda))$. Тогда согласно $(3.24)$ пара $(x(\xi, \lambda), u(\xi, \lambda))$ является решением управляемой системы $(1.1),(1.3)$. Воспользовавшись леммой 3.1 , мы получим, что для любой траектории $x(\xi, \lambda),(\xi, \lambda) \in Q$, системы $(1.1),(1.3)$ справедливо неравенство (3.1). Поэтому в соответствии с (2.6)

$$
\|\overline{\mathrm{co}} U(t, x(\xi, \lambda)(t), \xi)\|_{Y} \leqslant a_{K}(t)+b_{K}(t) M_{1}^{2 / q}
$$

где $K \subset \Lambda$ - проекция множества $Q$ на $\Lambda$ и $M_{1}$ - константа из неравенства (3.1).

Обозначим

$$
\mathscr{F}=\left\{\varphi \in L^{q}(T, Y):\|\varphi(t)\|_{Y} \leqslant a_{K}(t)+b_{K}(t) M_{1}^{2 / q} \text { п.в. }\right\}
$$

Тогда $\mathscr{F}$ является выпуклым, метризуемым, компактным подмножеством пространства $w-L^{q}(T, Y)$ и

$$
\operatorname{Fix} \overline{\operatorname{co}} \Phi(\xi, \lambda) \subset \mathscr{F}, \quad(\xi, \lambda) \in Q .
$$


Известно, что если значения многозначного отображения лежат в компакте, то полунепрерывность сверху по Вьеторису отображения и компактность его значений следуют из замкнутости его графика. Поэтому нам достаточно показать замкнутость в $\Lambda \times H \times w-L^{q}(T, Y)$ графика сужения отображения $\operatorname{Fix} \overline{c o} \Phi(\xi, \lambda)$ на $Q$.

Пусть $\left(\xi_{n}, \lambda_{n}\right) \rightarrow(\xi, \lambda)$ в $\Lambda \times H$ и $\varphi_{n} \rightarrow \varphi$ в $w-L^{q}(T, Y),\left(\xi_{n}, \lambda_{n}\right) \in Q, \varphi_{n} \in$ $\operatorname{Fix} \overline{\operatorname{co}} \Phi\left(\xi_{n}, \lambda_{n}\right), n \geqslant 1$. Поскольку $\varphi_{n} \in \overline{\operatorname{co}} \Phi\left(\xi_{n}, \lambda_{n}, \varphi_{n}\right), n \geqslant 1$, то из леммы 4.1 и теоремы Мазура следует, что

$$
\varphi \in \bigcap_{n \geqslant 1} \overline{\operatorname{co}}\left(\bigcup_{k \geqslant n} \varphi_{k}\right) \subset \bigcap_{n \geqslant 1} \overline{\operatorname{co}}\left(\bigcup_{k \geqslant n} \overline{\operatorname{co}} \Phi\left(\xi_{k}, \lambda_{k}, \varphi_{k}\right)\right) \subset \overline{\operatorname{co}} \Phi(\xi, \lambda, \varphi) .
$$

Следовательно, $\varphi \in \mathrm{Fix} \overline{\mathrm{co}} \Phi(\xi, \lambda)$. Воспользовавшись (5.5), мы получим, что значениями отображения $\operatorname{Fix} \Phi(\xi, \lambda)$ являются компактные множества из $w-L^{q}(T, Y)$ и отображение $\mathrm{Fix} \Phi(\xi, \lambda)$ полунепрерывно сверху по Вьеторису из $\Lambda \times H$ в $w-L^{q}(T, Y)$. Лемма доказана.

Рассмотрим теперь вопрос об аппроксимации непрерывных селекторов отображения $\operatorname{Fix} \overline{c o} \Phi(\xi, \lambda)$ непрерывными селекторами отображения $\operatorname{Fix} \overline{\operatorname{co}} \Phi(\xi, \lambda)$.

ТЕОРема 5.2. Пусть выполняются гипотезы $Г(U)$ 1)-3) и неравенство (2.6). Тогда для любого непрерывного селектора $v: \Lambda \times H \rightarrow L^{q}(T, Y)$ отображения $\operatorname{Fix} \overline{\operatorname{co}} \Phi(\xi, \lambda)$, любого $\varepsilon>0$ и любого компакта $Q \subset \Lambda \times H$ существует непрерывный селектор $u: \Lambda \times H \rightarrow L^{q}(T, Y)$ отображения $\operatorname{Fix} \Phi(\xi, \lambda)$ такой, чmo

$$
\|v(\xi, \lambda)-u(\xi, \lambda)\|_{\omega}<\varepsilon, \quad(\xi, \lambda) \in Q,
$$

əде $\|\cdot\|_{\omega}-$ норма $(5.1)$.

ДокАЗАТЕльство. Пусть $v: \Lambda \times H \rightarrow L^{q}(T, Y)$ - непрерывный селектор отображения $\mathrm{Fix} \overline{\mathrm{co}} \Phi(\xi, \lambda)$, который существует согласно лемме 5.1 , и $Q \subset \Lambda \times H-$ компактное множество. Тогда, как было показано при доказательстве леммы 5.1, имеет место включение $(5.5)$ с $\mathscr{F}$, определенным соотношением (5.4). Поэтому в соответствии с (5.3), (5.4)

$$
\overline{\mathrm{co}} \Phi(\xi, \lambda, v(\xi, \lambda)) \subset \mathscr{F}, \quad(\xi, \lambda) \in Q .
$$

Поскольку $\mathscr{F}$ является компактным, метризуемьм в $w$ - $L^{q}(T, Y)$ множеством, то в соответствии с леммой 4.1 сужение отображения $\Phi(\xi, \lambda, u)$ на $Q \times \mathscr{F}$ является непрерывным из $\Lambda \times H \times w-L^{q}(T, Y)$ в $\left(\operatorname{cb} L^{q}(T, Y), D_{L}(\cdot, \cdot)\right)$. Так как $\mathscr{F}$ является разложимым, замкнутым, ограниченным подмножеством пространства $L^{q}(T, Y)$, то утверждение теоремы вытекает из [13; теорема 5.1]. Теорема доказана.

\section{§6. Основные результаты}

В этом параграфе мы приведем основные результаты нашей работы.

ТеОРема 6.1. Пусть выполняются гипотезъ $Г(U)$ 1)-4). Тогда для любъх $(\xi, \lambda)$ множсество $\mathscr{R}_{U}(\xi, \lambda)$ не пусто и существует непрерывная функиия $s: \Lambda \times H \rightarrow C(T, H) \times L^{q}(T, Y)$ такая, что

а) справедливо

$$
s(\xi, \lambda) \in \mathscr{R}_{U}(\xi, \lambda), \quad(\xi, \lambda) \in \Lambda \times H
$$


b) если $D \subset \Lambda \times H-$ замкнутое множество и $s_{D}: D \rightarrow C(T, H) \times L^{q}(T, Y)$ - непрерывная функция, $s_{D}(\xi, \lambda) \in \mathscr{R}_{U}(\xi, \lambda),(\xi, \lambda) \in D$, то существует непрерывная функция $s: \Lambda \times H \rightarrow C(T, H) \times L^{q}(T, Y)$ такая, что справедливо включение (6.1) и $s(\xi, \lambda)=s_{D}(\xi, \lambda),(\xi, \lambda) \in D$.

СлЕДСТВИЕ 6.1. Пусть выполняются предположения теоремы 6.1. Тогда

а) отображсение $(\xi, \lambda) \mapsto \mathscr{R}_{U}(\xi, \lambda)$ является полунепрерьвным снизу по Вветорису из $\Lambda \times H$ в $C(T, H) \times L^{q}(T, Y)$ с замкнутыми в $C(T, H) \times$ $L^{q}(T, Y)$ значениями;

b) для любых $(\xi, \lambda)$ множество $\mathscr{R}_{U}(\xi, \lambda)$ является абсолютным ретрактом в $C(T, H) \times L^{q}(T, Y)$, и ретракцию можно выбрать непрерьвно зависящей от $(\xi, \lambda)$, т.е. существует непрерьвная функция $g: \Lambda \times H \times$ $C(T, H) \times L^{q}(T, Y) \rightarrow C(T, H) \times L^{q}(T, Y)$ maкая, ито

$$
\begin{gathered}
g(\xi, \lambda, x, u) \in \mathscr{R}_{U}(\xi, \lambda), \quad(\xi, \lambda, x, u) \in \Lambda \times H \times C(T, H) \times L^{q}(T, Y), \\
g(\xi, \lambda, x, u)=(x, u), \quad(x, u) \in \mathscr{R}_{U}(\xi, \lambda), \quad(\xi, \lambda) \in \Lambda \times H
\end{gathered}
$$

с) если пространство $\Lambda$ дугообразно связно, то множество

$$
\left\{\bigcup_{(\xi, \lambda) \in \Lambda \times H} \mathscr{R}_{U}(\xi, \lambda)\right\}
$$

дугообразно связно;

d) для любых непрерывных селекторов $s_{i}: \Lambda \times H \rightarrow C(T, H) \times L^{q}(T, Y), i=$ 1,2 , отображсения $\mathscr{R}_{U}(\xi, \lambda)$ существует непрерывное отображение $h: \Lambda \times H \times[0,1] \rightarrow C(T, H) \times L^{q}(T, Y)$ такое, ито

$$
\begin{gathered}
h(\xi, \lambda, 0)=s_{1}(\xi, \lambda), \quad h(\xi, \lambda, 1)=s_{2}(\xi, \lambda), \quad(\xi, \lambda) \in \Lambda \times H, \\
h(\xi, \lambda, \tau) \in \mathscr{R}_{U}(\xi, \lambda), \quad(\xi, \lambda, \tau) \in \Lambda \times H \times[0,1] .
\end{gathered}
$$

Утверждение d) следствия 6.1 означает, что любые два непрерывных селектора отображения $(\xi, \lambda) \mapsto \mathscr{R}_{U}(\xi, \lambda)$ можно соединить гомотопией со значениями в $\mathscr{R}_{U}(\xi, \lambda)$.

Если $(x(\xi, \lambda), u(\xi, \lambda))$ - решение управляемой системы $(1.1),(1.2)$, то в соответствии с определением решения и (3.24), (4.1)

$$
\begin{aligned}
x(\xi, \lambda) & =\mathscr{T}(\xi, \lambda, u(\xi, \lambda)), \\
u(\xi, \lambda) & \in \Phi(\xi, \lambda, u(\xi, \lambda)) .
\end{aligned}
$$

Поэтому пара $(x(\xi, \lambda), u(\xi, \lambda))$ является решением управляемой системы $(1.1),(1.2)$ тогда и только тогда, когда справедливы соотношения (6.2), (6.3). Включение (6.3) эквивалентно тому, что $u(\xi, \lambda) \in \operatorname{Fix} \Phi(\xi, \lambda)$. Поэтому если $u(\xi, \lambda) \in \Lambda \times H \rightarrow$ $L^{q}(T, Y)$ является непрерывным селектором отображения $\operatorname{Fix} \Phi(\xi, \lambda)$, то $x(\xi, \lambda)$, определенное соотношением (6.2), согласно теореме 3.1 является непрерывным отображением из $\Lambda \times H$ в $C(T, H)$. Тогда функция $s(\xi, \lambda)=(x(\xi, \lambda), u(\xi, \lambda))$ является непрерывным селектором отображения $\mathscr{R}_{U}(\xi, \lambda)$. Поэтому с учетом теоремы 3.1 утверждения теоремы 6.1, утверждения а), c), d) и первая часть утверждения b) 
следствия 6.1 являются перефразировками подобных утверждений из теоремы 5.1 и следствия 5.1 .

Докажем теперь вторую часть утверждения b) следствия 6.1. Пусть $Z=$ $C(T, H) \times L^{q}(T, Y)$. Пространство $Z$ является метрическими и сепарабельным. Рассмотрим многозначное отображение $\Phi^{*}: \Lambda \times H \times Z \times L^{q}(T, Y) \rightarrow L^{q}(T, Y)$, определенное по правилу

$$
\Phi^{*}(\xi, \lambda, z, u)=\Phi(\xi, \lambda, u), \quad(\xi, \lambda, u) \in \Lambda \times H \times L^{q}(T, Y), \quad z \in Z .
$$

Рассматривая $z \in Z$ как еше один новый параметр, мы получаем, что многозначное отображение $\Phi^{*}(\xi, \lambda, z, u)$ обладает теми же свойствами 1$\left.\left.), 2\right), 4\right)$, которые установлены для отображения $\Phi(\xi, \lambda, u)$ в теореме 4.1. Поэтому для отображения $\operatorname{Fix} \Phi^{*}(\xi, \lambda, z)$ согласно [12; теорема 3.1$]$ справедливы утверждения теоремы 5.1. Обозначим через $D$ график отображения $\mathscr{R}_{U}(\xi, \lambda)$.

Воспользовавшись утверждением 4) теоремы 4.1, теоремой 3.1 и (6.2), (6.3), мы получим, что $D$ является замкнутым подмножеством пространства $\Lambda \times H \times Z$.

Пусть $z \in Z$, тогда $z=(x, u), x \in C(T, H), u \in L^{q}(T, Y)$. Рассмотрим функцию $h_{D}: D \rightarrow L^{q}(T, Y)$, определенную следуюшим образом:

$$
h_{D}(\xi, \lambda, z)=u, \quad(\xi, \lambda, z) \in D .
$$

Эта функция непрерывна на множестве $D$ и, как следует из (6.3), (6.5),

$$
h_{D}(\xi, \lambda, z) \in \Phi(\xi, \lambda, u), \quad(\xi, \lambda, z) \in D .
$$

Воспользовавшись (6.4)-(6.6), мы получим

$$
h_{D}(\xi, \lambda, z) \in \Phi\left(\xi, \lambda, h_{D}(\xi, \lambda, z)\right)=\Phi^{*}\left(\xi, \lambda, z, h_{D}(\xi, \lambda, z)\right), \quad(\xi, \lambda, z) \in D
$$

Из (6.7) следует, что $h_{D}(\xi, \lambda, z)$ является непрерьвным селектором отображения $\operatorname{Fix} \Phi^{*}(\xi, \lambda, z)$, определенным на $D$. Воспользовавшись утверждением b) теоремы 5.1 , примененным к отображению $\operatorname{Fix} \Phi^{*}(\xi, \lambda, z)$, мы получим, что сушествует непрерывная функция $h: \Lambda \times H \times Z \rightarrow L^{q}(T, Y)$ такая, что

$$
\begin{gathered}
h(\xi, \lambda, z) \in \operatorname{Fix} \Phi^{*}(\xi, \lambda, z), \quad(\xi, \lambda, z) \in \Lambda \times H \times Z, \\
h(\xi, \lambda, z)=h_{D}(\xi, \lambda, z)=u, \quad(\xi, \lambda, z) \in D .
\end{gathered}
$$

Из (6.4), (6.8) следует, что

$$
h(\xi, \lambda, z) \in \Phi^{*}(\xi, \lambda, z, h(\xi, \lambda, z))=\Phi(\xi, \lambda, h(\xi, \lambda, z)) .
$$

Положим $x(\xi, \lambda, z)=\mathscr{T}(\xi, \lambda, h(\xi, \lambda, z))$. Из непрерывности $h: \Lambda \times H \times Z \rightarrow$ $L^{q}(T, Y)$ и теоремы 3.1 следует, что функция $x: \Lambda \times H \times Z \rightarrow C(T, H)$ непрерывна. Тогда согласно $(6.2),(6.3)$ и (6.10) пара $(x(\xi, \lambda, z), u(\xi, \lambda, z))$ является решением управляемой системы (1.1), (1.2). Положим $g(\xi, \lambda, z)=g(\xi, \lambda, x, u)=$ $(x(\xi, \lambda, x, u), h(\xi, \lambda, x, u))$, где $z=(x, u)$. Тогда $g(\xi, \lambda, x, u) \in \mathscr{R}_{U}(\xi, \lambda),(\xi, \lambda, x, u) \in$ $\Lambda \times H \times C(T, H) \times L^{q}(T, Y)$, и согласно $(6.9)$

$$
g(\xi, \lambda, x, u)=(x, u), \quad(x, u) \in \mathscr{R}_{U}(\xi, \lambda), \quad(\xi, \lambda) \in \Lambda \times H
$$

Вторая часть утверждения b) следствия 6.1 доказана. 
ЛЕмма 6.1. Пусть выполняются гипотезы $Г(U)$ 1)-3) и неравенство (2.6). Тогда для отображений $\mathscr{R}_{U}(\xi, \lambda)$ и $\mathscr{R}_{\overline{\mathrm{co}} U}(\xi, \lambda)$ справедливы все утвержсдения теоремы 6.1 и следствия 6.1. Более того, отображение $(\xi, \lambda) \mapsto \mathscr{R}_{\overline{\mathrm{co}} U}(\xi, \lambda)$ является непрерьвным по Вветорису из $\Lambda \times H$ в $C(T, H) \times w-L^{q}(T, Y)$ с компактными значениями в $C(T, H) \times w-L^{q}(T, Y)$.

ДокАЗАТЕльство. Первая часть утверждений леммы следует из леммы 5.1. Согласно утверждению а) теоремы 6.1 отображение $(\xi, \lambda) \mapsto \mathscr{R}_{\overline{\mathrm{co}}} U(\xi, \lambda)$ является полунепрерывным снизу по Вьеторису из $\Lambda \times H$ в $C(T, H) \times L^{q}(T, Y)$. Поэтому оно является полунепрерывным снизу по Вьеторису из $\Lambda \times H$ в $C(T, H) \times w-L^{q}(T, Y)$. Как и при доказательстве леммы 5.1 , нам осталось доказать, что сужение отображения $\mathscr{R}_{\overline{\mathrm{co}} U}(\xi, \lambda)$ на любой компакт $Q \subset \Lambda \times H$ является полунепрерывным сверху по Вьеторису из $\Lambda \times H$ в $C(T, H) \times w-L^{q}(T, Y)$ с компактными значениями в $C(T, H) \times w-L^{q}(T, Y)$.

Пусть $Q \subset \Lambda \times H-$ компакт. Тогда согласно лемме 5.1 график сужения отображения $\operatorname{Fix} \overline{\operatorname{co}} \Phi(\xi, \lambda)$ на $Q$ является компактомв $\Lambda \times H \times w-L^{q}(T, Y)$. Воспользовавшись теоремой 3.1, мы получим, что график сужения отображения $\mathscr{R}_{\overline{\mathrm{co}}} U(\xi, \lambda)$ на $Q$ является компактом в $\Lambda \times H \times C(T, H) \times w-L^{q}(T, Y)$. Но это означает, что сужение отображения $\mathscr{R} \overline{\overline{c o}} U(\xi, \lambda)$ на $Q$ является полунепрерывным сверху по Вьеторису из $\Lambda \times H$ в $C(T, H) \times w-L^{q}(T, Y)$ с компактными значениями в $C(T, H) \times w-L^{q}(T, Y)$. Лемма доказана.

Рассмотрим теперь вопрос об аппроксимации непрерывных селекторов отображения $\mathscr{R}_{\overline{\mathrm{co}} U}(\xi, \lambda)$ непрерывными селекторами отображения $\mathscr{R}_{U}(\xi, \lambda)$. Рассмотрим пространство $C(T, H) \times L_{\omega}^{q}(T, Y)($ см. $(5.1))$ с нормой

$$
\|h\|_{C, \omega}=\|(x, u)\|_{C, \omega}=\|x\|_{C(T, H)}+\|u\|_{\omega}
$$

где $\|\cdot\|_{\omega}-$ норма $(5.1), h=(x, u) \in C(T, H) \times L_{w}^{q}(T, Y)$.

ТЕОРема 6.2. Пусть выполняются гипотезы $\Gamma(U)$ 1)-3) и неравенство (2.6). Тогда для любого непрерывного селектора $g: \Lambda \times H \rightarrow C(T, H) \times L^{q}(T, Y)$ отображсения $\mathscr{R}_{\overline{\mathrm{co}} U}(\xi, \lambda)$, любого $\varepsilon>0$ и любого компакта $Q \subset \Lambda \times H$ существует непрерывный селектор $h: \Lambda \times H \rightarrow C(T, H) \times L^{q}(T, Y)$ отображсения $\mathscr{R}_{U}(\xi, \lambda)$ mакой, что

$$
\|g(\xi, \lambda)-h(\xi, \lambda)\|_{C, \omega}<\varepsilon, \quad(\xi, \lambda) \in Q
$$

ДоКАЗАТЕЛЬСтво. Возьмем произвольный компакт $Q \subset \Lambda \times H$, и пусть $g: \Lambda \times H \rightarrow C(T, H) \times L^{q}(T, Y)$ - непрерьвньй селектор отображения $\mathscr{R}_{\overline{\operatorname{co}} U}(\xi, \lambda)$, которьй существует согласно лемме 6.1. Тогда $g(\xi, \lambda)=(x(\xi, \lambda), v(\xi, \lambda))$, где $x(\xi, \lambda)=\mathscr{T}(\xi, \lambda, v(\xi, \lambda))$ и $v(\xi, \lambda)$ - непрерывный селектор отображения Fix $\overline{c o} \Phi(\xi, \lambda)$. Как было показано при доказательстве теоремы 5.2, существует вьпуклое, метризуемое, компактное подмножество $\mathscr{F} \subset w-L^{q}(T, Y)$ такое, что справедливо включение (5.5). Согласно теореме 5.2 сушествует последовательность $v_{n}: \Lambda \times H \rightarrow L^{q}(T, Y)$ непрерывных селекторов отображения $\operatorname{Fix} \Phi(\xi, \lambda)$ такая, что

$$
\lim _{n \rightarrow \infty} \sup _{(\xi, \lambda) \in Q}\left\|v(\xi, \lambda)-v_{n}(\xi, \lambda)\right\|_{\omega}=0
$$


Положим

$$
Z=\bigcup_{(\xi, \lambda) \in Q}\left(v(\xi, \lambda) \cup\left(\bigcup_{n \geqslant 1} v_{n}(\xi, \lambda)\right)\right) \subset L^{q}(T, Y) .
$$

Так как $v_{n}(\xi, \lambda) \in \operatorname{Fix} \overline{c o} \Phi(\xi, \lambda), n \geqslant 1$, то в соответствии с $(5.5)$

$$
Z \subset \mathscr{F}
$$

Так как любое непрерывное отображение из $\Lambda \times H$ в $L^{q}(T, Y)$ является непрерывньм и из $\Lambda \times H$ в $L_{\omega}^{q}(T, Y)$, то из (6.13) следует, что множество $Z$ является компактом в $L_{\omega}^{q}(T, Y)$. Пусть $Z_{\omega}-$ множество с топологией, индуцированной топологией пространства $L_{\omega}^{q}(T, Y)$. В соответствии с (6.15) $Z$ является ограниченным подмножеством пространства $L^{q}(T, Y)$. Тогда из [13; лемма 5.1] вытекает, что на множестве $Z$ топологии пространств $w-L^{q}(T, Y)$ и $L_{\omega}^{q}(T, Y)$ совпадают. Поэтому в соответствии с теоремой 3.1 отображение $\mathscr{T}: Q \times Z_{\omega} \rightarrow C(T, H)$ является непрерывным. Поскольку множество $Q \times Z_{\omega}$ является компактом, то отображение $\mathscr{T}: Q \times Z_{\omega} \rightarrow C(T, H)$ равномерно непрерывно.

Пусть $x_{n}(\xi, \lambda)=\mathscr{T}\left(\xi, \lambda, v_{n}(\xi, \lambda)\right), n \geqslant 1$. Тогда $g_{n}(\xi, \lambda)=\left(x_{n}(\xi, \lambda), v_{n}(\xi, \lambda)\right)$, $n \geqslant 1$, является непрерьвньм селектором отображения $\mathscr{R}_{U}(\xi, \lambda)$. Воспользовавшись $(6.13),(6.14)$ и равномерной непрерывностью отображения $\mathscr{T}: Q \times Z_{\omega} \rightarrow$ $C(T, H)$, мы получим, что

$$
\lim _{n \rightarrow \infty} \sup _{(\xi, \lambda) \in Q}\left\|x_{n}(\xi, \lambda)-x(\xi, \lambda)\right\|_{C(T, H)}=0
$$

Поэтому в соответствии с $(6.13),(6.16)$ для $\varepsilon>0$ сушествует $n_{0}$ такое, что

$$
\left\|v(\xi, \lambda)-v_{n}(\xi, \lambda)\right\|_{\omega}<\frac{\varepsilon}{2}, \quad\left\|x(\xi, \lambda)-x_{n}(\xi, \lambda)\right\|_{C(T, H)}<\frac{\varepsilon}{2}
$$

$n \geqslant n_{0},(\xi, \lambda) \in Q$. Из (6.17) и (6.11) следует, что

$$
\left\|g(\xi, \lambda)-g_{n}(\xi, \lambda)\right\|_{C, \omega}<\varepsilon, \quad(\xi, \lambda) \in Q, \quad n \geqslant n_{0} .
$$

Теперь утверждение теоремы вытекает из последнего неравенства. Теорема доказана.

ЗАмечАнИЕ 6.1. Мы ввели гипотезу $\Gamma(U) 2$ ), чтобы в рамках единых предположений доказать все результаты, приведенные в статье. Ряд из них справедлив при более слабых предположениях, чем гипотеза $\Gamma(U) 2)$. Например, теоремы 4.1, $5.1,6.1$ и ряд утверждений из следствий 5.1, 6.1 остаются справедливьми, если вместо гипотезы $\Gamma(U) 2)$ предположить, что при почти всех $t \in T$ и любом $x \in H$ отображение $\xi \mapsto U(t, x, \xi)$ полунепрерьвно снизу по Вьеторису (детали см. в [7], $[12],[13])$.

ЗАмечАниЕ 6.2. Аппроксимация непрерывных селекторов отображения $\mathscr{R}_{\overline{\mathrm{co}} U}(\xi, \lambda)$ непрерывными селекторами отображения $\mathscr{R}_{U}(\xi, \lambda)$ обычно называется релаксацией. Поэтому теорема 6.2 является теоремой релаксации для селекторов решений управляемой системы. 


\section{§7. Пример}

В этом параграфе мы рассмотрим пример управляемой параболической системы и дадим интерпретацию полученных вьше абстрактных результатов.

Пусть $T=[0,1], \Lambda$ - компактное метрическое пространство, $Z$ - ограниченная область в $\mathbb{R}^{N}$ с гладкой границей $\partial Z$ и $Z_{1}$ - проекция $Z$ на первую координатную ось пространства $\mathbb{R}^{N}$. Рассмотрим управляемую систему, описываемую параболическим уравнением

$$
\begin{gathered}
\frac{\partial x}{\partial t}-\sum_{i, j=1}^{N} D_{i}\left(a_{i j}\left(t, z_{1}, \xi\right) D_{j} x\right)=f(t, z) u(t, z), \\
\left.x\right|_{T \times \partial Z}=0, \quad x(0, z)=x_{0}(z)=\lambda(z) \\
u(t, z) \in \widetilde{U}(t, z, x(t, z), \xi) \text { п.в. в } T \times Z .
\end{gathered}
$$

Здесь $D_{i}=\partial / \partial z_{i}, i=1, \ldots, N$.

Наряду с ограничениями (7.2) мы рассмотрим ограничение

$$
u(t, z) \in \operatorname{co} \widetilde{U}(t, z, x(t, z), \xi) \text { п.в. в } T \times Z \text {. }
$$

Введем следующие предположения, которые всюду в дальнейшем считаем выполненными.

ПреДПОЛОЖЕНИЯ Г $(a)$. Считаем, что $a_{i j}\left(t, z_{1}, \xi\right)=a_{j i}\left(t, z_{1}, \xi\right), i=1, \ldots, N$, $z_{1} \in Z_{1}, \xi \in \Lambda$, являются элементами пространства $L^{\infty}\left(T \times Z_{1}\right)$ такими, что

1) существуют константы $0<m<M$, для которых имеют место неравенства

$$
m|z|^{2} \leqslant \sum_{i, j=1}^{N} a_{i j}\left(t, z_{1}, \xi\right) z_{i} z_{j} \leqslant M|z|^{2}, \quad z \in Z, \quad \xi \in \Lambda, \quad t \in T
$$

2) сушествует функция $l(t, z) \in L^{\infty}(T \times Z)$ такая, что

$$
\left|a_{i j}\left(t, z_{1}, \xi\right)-a_{i j}\left(t+\tau, z_{1}, \xi\right)\right| \leqslant l(t, z)|\tau|, \quad z \in Z, \quad \xi \in \Lambda, \quad t+\tau, t \in T
$$

3) если $\xi_{n} \rightarrow \xi$, то для любого $t \in T$

$$
\begin{gathered}
\frac{1}{a_{11}\left(t, \cdot, \xi_{n}\right)} \rightarrow \frac{1}{a_{11}(t, \cdot, \xi)}, \quad \frac{a_{i 1}\left(t, \cdot, \xi_{n}\right)}{a_{11}\left(t, \cdot, \xi_{n}\right)} \rightarrow \frac{a_{i 1}(t, \cdot, \xi)}{a_{11}(t, \cdot, \xi)}, i=2, \ldots, N \\
a_{i j}\left(t, \cdot, \xi_{n}\right)-\frac{a_{i 1}\left(t, \cdot, \xi_{n}\right) a_{1 j}\left(t, \cdot, \xi_{n}\right)}{a_{11}\left(t, \cdot, \xi_{n}\right)} \rightarrow a_{i j}(t, \cdot, \xi)-\frac{a_{i 1}(t, \cdot, \xi) a_{1 j}(t, \cdot, \xi)}{a_{11}(t, \cdot, \xi)} \\
i, j=1, \ldots, N, \quad i \neq 1, \quad j \neq 1,
\end{gathered}
$$

где сходимость понимается в $\sigma\left(L^{\infty}(Z), L^{1}(Z)\right)$ топологии.

ПРЕДПОЛОЖЕНИЕ $Г(f)$. Функция $f(t, z)$ является элементом пространства $L^{\infty}(T \times Z)$. 
ПреДПоЛоЖЕния $Г(\widetilde{U})$. Считаем, что $\widetilde{U}: T \times Z \times R \times \Lambda \rightarrow R$ является многозначным отображением с замкнутыми значениями таким, что

1) отображение $(t, z) \mapsto \widetilde{U}(t, z, x, \xi)$ измеримо;

2) отображение $\xi \mapsto \widetilde{U}(t, z, x, \xi)$ непрерьвно п.в. на $T \times Z$ для любого $x \in \mathbb{R}$;

3) существует непрерывная функция $l: \Lambda \rightarrow L^{2}\left(T, \mathbb{R}^{+}\right)$такая, что для любых $x, y \in \mathbb{R}, \xi \in \Lambda$ почти всюду на $T \times Z$

$$
D_{\mathbb{R}}(\widetilde{U}(t, z, x, \xi), \widetilde{U}(t, z, y, \xi)) \leqslant l(\xi)(t)|x-y|
$$

4) существуют $a(t, z) \in L^{2}\left(T \times Z, \mathbb{R}^{+}\right)$и $b>0$ такие, что $|U(t, z, x, \xi)| \leqslant$ $a(t, z)+b|x|$ п.в. на $T \times Z$ для любых $x \in \mathbb{R}, \xi \in \Lambda$.

ПРЕДПОЛОЖЕНИЕ $Г\left(x_{0}\right)$. Считаем, что $x_{0}=\lambda \in L^{2}(Z)$.

Всюду в дальнейшем мы считаем, что гипотезы $\Gamma(a), \Gamma(f), \Gamma(\widetilde{U}), \Gamma\left(x_{0}\right)$ имеют место.

ЗАмЕчАниЕ 7.1. Так как в рамках гипотез $\Gamma(\widetilde{U})$ значениями отображения $\widetilde{U}(t, z, x, \lambda)$ являются компактные подмножества пространства $\mathbb{R}$, то $\overline{\mathrm{co}} \widetilde{U}(t, z, x, \lambda)=\operatorname{co} \widetilde{U}(t, z, x, \lambda)$, где со означает выпуклую оболочку множества. Поэтому вместо ограничения (1.3) мы рассматриваем ограничение 7.3.

В нашем примере мы берем $p=2$.

Пусть $H_{0}^{1}(Z)$ - пространство Соболева [17]. Тогда его сопряженным является пространство $H^{-1}(Z)$. Положим $V=H_{0}^{1}(Z), H=L^{2}(Z), V^{*}=H^{-1}(Z)$. В качестве пространства $Y$ мы берем пространство $L^{2}(Z)$. Как обычно, пространство $L^{2}\left(T, L^{2}(Z)\right)$ мы отождествляем с пространством $L^{2}(T \times Z)$, полагая $u(t)(z)=u(t, z)$ для элемента $u \in L^{2}\left(T, L^{2}(Z)\right)$ и $v(t, z)=v(t)(z)$ для элемента $v \in L^{2}(T \times Z)$.

ОПРЕДЕЛЕНИЕ 7.1. При фиксированных $\xi \in \Lambda, \lambda \in L^{2}(Z)$ пара функций $(x(\xi, \lambda), u(\xi, \lambda)), x(\xi, \lambda) \in L^{2}\left(T, H_{0}^{1}(Z)\right)$ с $d x / d t \in L^{2}\left(T, H^{-1}(Z)\right)$ и $u \in L^{2}(T \times Z)$ $=L^{2}\left(T, L^{2}(Z)\right)$, называется решением управляемой системы (7.1), (7.2), если для почти всех $t \in T$ и всех $y \in H_{0}^{1}(Z)$ справедливы равенства

$$
\begin{gathered}
\frac{d}{d t} \int_{Z} x(t, z) y(z) d z+\int_{Z} \sum_{i, j=1}^{N} a_{i j}\left(t, z_{1}, \xi\right) D_{i} x D_{j} y d z=\int_{Z} f(t, z) u(t, z) y(z) d z \\
x(0, z)=\lambda(z)
\end{gathered}
$$

и включение

$$
u(t, z) \in U(t, z, x(t, z), \xi) \text { п.в. на } T \times Z \text {. }
$$

Сведем управляемую систему (7.1), (7.2) к управляемой системе (1.1), (1.2). Рассмотрим форму Дирихле $a: T \times H_{0}^{1}(Z) \times H_{0}^{1}(Z) \times \Lambda \rightarrow \mathbb{R}$, определенную по формуле

$$
a(t, \xi, x, y)=\int_{Z} \sum_{i, j} a_{i j}\left(t, z_{1}, \xi\right) D_{i} x D_{j} y d z, \quad x, y \in H_{0}^{1}(Z)
$$


Воспользовавшись гипотезой $\Gamma(a)$ 1), с помощю хорошо известных рассуждений получим, что существует оператор $A: T \times H_{0}^{1}(Z) \times \Lambda \rightarrow H^{-1}(Z)$ такой, что

$$
\langle y, A(t, x, \xi)\rangle=a(t, \xi, x, y), \quad x, y \in H_{0}^{1}(Z) .
$$

При фиксированных $t \in T, \xi \in \Lambda$ этот оператор является линейным, непрерывным и удовлетворяет гипотезам $\Gamma(A) 1)-4)$ с $K=\Lambda$.

Из гипотезы $\Gamma(a) 2)$ с достаточной очевидностью вытекает гипотеза $\Gamma(A) 5)$. И, наконец, из гипотезы $\Gamma(a) 3)$ и [23; лемма 3$]$ мы получаем, что оператор $A(t, x, \xi)$ удовлетворяет гипотезе $\Gamma(A) 6)$. Таким образом, оператор $A(t, x, \xi)$, определенньй по формуле (7.4), удовлетворяет всем гипотезам $Г(A)$.

Пусть $B(t) u, u \in L^{2}(Z),-$ функция, определенная по правилу

$$
(B(t) u)(z)=f(t, z) u(z), \quad z \in Z .
$$

Воспользовавшись гипотезой $\Gamma(f)$ и рассуждениями, приведенньми в $[24 ; \S 7]$, мы получим, что формула (7.5) определяет оператор $B: T \rightarrow \mathscr{L}\left(L_{2}(Z), L_{2}(Z)\right)$, удовлетворяющий гипотезам $\Gamma(B)$.

Рассмотрим многозначное отображение $(t, x) \mapsto U(t, x, \xi), x \in L^{2}(Z), \xi \in \Lambda$, определенное по правилу

$$
U(t, x, \xi)=\left\{u \in L^{2}(Z): u(z) \in \widetilde{U}(t, z, x(z), \xi) \text { п.в. на } Z\right\} .
$$

Согласно [19; теорема 1.5] имеет место равенство

$$
\overline{\mathrm{co}} U(t, x, \xi)=\left\{u \in L^{2}(Z): u(z) \in \operatorname{co} \widetilde{U}(t, z, x(z), \xi) \text { п.в. на } Z\right\} .
$$

Как было показано в [24; $\S]$, при вьполнении гипотез $Г(\widetilde{U}) 1), 3), 4)$ отображение $U(t, x, \xi)$ является отображением из $T \times L^{2}(Z) \times \Lambda$ в $\mathrm{c} L^{2}(Z)$ и удовлетворяет гипотезам $\Gamma(U) 1), 3)$ и неравенству (2.6). Так как при фиксированном $x \in L^{2}(Z)$ при почти каждом $t \in T$ отображение $z \mapsto \widetilde{U}(t, z, x(z), \xi)$ измеримо [24; 7$]$, то по аналогии с (3.5), принимая во внимание (7.6), мы получим

$D_{L^{2}(Z)}\left(U\left(t, x, \xi_{1}\right), U\left(t, x, \xi_{2}\right)\right) \leqslant\left(\int_{Z} D_{\mathbb{R}}\left(\widetilde{U}\left(t, z, x(z), \xi_{1}\right), \widetilde{U}\left(t, z, x(z), \xi_{2}\right)\right)^{2} d z\right)^{1 / 2}$.

Воспользовавшись гипотезами $\Gamma(\widetilde{U}) 3), 4),(7.8)$ и теоремой Лебега об ограниченной сходимости, мы приходим к выводу, что при почти всех $t \in T$ для любого $x \in L^{2}(Z)$ отображение $\xi \mapsto U(t, x, \xi)$ непрерывно, т.е. удовлетворяет гипотезе $\Gamma(U) 2)$. Следовательно, отображение $U(t, x, \xi)$ удовлетворяет гипотезам $\Gamma(U)$ 1)-3) и неравенству (2.6).

И, наконец, включение $u(t) \in U(t, x(t), \xi), x \in C\left(T, L^{2}(Z)\right), u \in L^{2}\left(T, L^{2}(Z)\right)=$ $L^{2}(T \times Z)$, имеет место почти всюду на $T$ тогда и только тогда, когда $u(t, z)=$ $u(t)(z) \in \widetilde{U}(t, z, x(t, z), \xi)$ почти всюду на $T \times Z[24 ; \S 7]$. Поэтому в соответствии с (7.2), (7.3) и (7.6), (7.7) с точки зрения определения решений управляемые системы (7.1), (7.2) и (7.1), (7.3) и порожденные ими управляемые системы (1.1), $(1.2)$ и $(1.1),(1.3)$ с операторами $A, B$ и многозначными отображениями $U(t, x, \xi)$ и $\overline{\mathrm{co}} U(t, x, \xi)$, определенными соотношениями $(7.4),(7.5),(7.6)$ и $(7.7)$, эквивалентны, т.е. имеют одни и те же решения. Поэтому применительно к управляемьм системам (7.1), (7.2) и (7.1), (7.3) мы можем сформулировать все результаты $\S 6$. Приведем некоторые из них.

Через $\mathscr{R}_{\widetilde{U}}(\xi, \lambda) \subset C\left(T, L^{2}(Z)\right) \times L^{2}(T \times Z), \xi \in \Lambda, \lambda \in L^{2}(Z)$, обозначим множество решений управляемой системы $(7.1),(7.2)$. Под $\mathscr{R}{ }_{\text {со } \widetilde{U}}(\xi, \lambda)$ мы понимаем множество решений управляемой системы $(7.1),(7.3)$. 
Teорема 7.1. Для любых $(\xi, \lambda) \in \Lambda \times L^{2}(Z)$ множество $\mathscr{R}_{\widetilde{U}}(\xi, \lambda) \subset$ $C\left(T, L^{2}(Z)\right) \times L^{2}(T \times Z)$ не пусто и существует непрерьвная функиия $s: \Lambda \times L^{2}(Z) \rightarrow C\left(T, L^{2}(Z)\right) \times L^{2}(T \times Z)$ maкая, чmo

а) справедливо

$$
s(\xi, \lambda) \in \mathscr{R}_{\widetilde{U}}(\xi, \lambda), \quad(\xi, \lambda) \in \Lambda \times H
$$

b) если $D \subset \Lambda \times L^{2}(Z)$ - замкнутое множество и $s_{D}: D \rightarrow C\left(T, L^{2}(Z)\right) \times$ $L^{2}(T \times Z)$ - непрерьвная функция, $s_{D}(\xi, \lambda) \in \mathscr{R}_{\widetilde{U}}(\xi, \lambda),(\xi, \lambda) \in D$, то существует непрерывная функция $s: \Lambda \times L^{2}(Z) \rightarrow C\left(T, L^{2}(Z)\right) \times$ $L^{2}(T \times Z)$ такая, что справедливо включение $(7.9)$ и $s(\xi, \lambda)=s_{D}(\xi, \lambda)$, $(\xi, \lambda) \in D$

c) отображение $(\xi, \lambda) \mapsto \mathscr{R}_{\widetilde{U}}(\xi, \lambda)$ является полунепрерывныцм снизу по Вветорису из $\Lambda \times L^{2}(Z)$ в $C\left(T, L^{2}(Z)\right) \times L^{2}(T \times Z)$ с замкнутыми значениями, а отображение $(\xi, \lambda) \mapsto \mathscr{R}_{\text {со } \widetilde{U}}(\xi, \lambda)$ является непрерывным по Вветорису из $\Lambda \times L^{2}(Z)$ в $C\left(T, L^{2}(Z)\right) \times w-L^{2}(T \times Z)$ с компактныцми значениями.

TЕОРема 7.2. Для любого непрерывного селектора $g=(x, u): \Lambda \times L^{2}(Z) \rightarrow$ $C\left(T, L^{2}(Z)\right) \times L^{2}(T \times Z)$ отображения $\mathscr{R}_{\text {со } \widetilde{U}}(\xi, \lambda)$, любого $\varepsilon>0$ и любого компакта $Q \subset \Lambda \times L^{2}(Z)$ существует непрерывный селектор $h=(y, v): \Lambda \times$ $L^{2}(Z) \rightarrow C\left(T, L^{2}(Z)\right) \times L^{2}(T \times Z)$ отображения $\mathscr{R}_{\widetilde{U}}(\xi, \lambda)$ такой, что

$$
\begin{gathered}
\sup _{t \in T}\left(\int_{\Omega}|x(\xi, \lambda)(t, z)-y(\xi, \lambda)(t, z)|^{2} d z\right)^{1 / 2}<\varepsilon, \quad(\xi, \lambda) \in Q, \\
\sup _{0 \leqslant t^{\prime} \leqslant t \leqslant 1}\left(\int_{\Omega}\left|\int_{t^{\prime}}^{t}(u(\xi, \lambda)(\tau, z)-v(\xi, \lambda)(\tau, z)) d \tau\right|^{2} d z\right)^{1 / 2}<\varepsilon, \quad(\xi, \lambda) \in Q .
\end{gathered}
$$

Теорема 7.1 представляет собой перефразировку аналогичных утверждений из теоремы 6.1, следствия 6.1 и леммы 6.1. Теорема 7.2 является аналогом теоремы 6.2. Что же касается неравенств (7.10), (7.11), то они вытекают из (6.11) и (5.1). Мы не приводим других аналогов остальных результатов из $\S 6$ ввиду их очевидности, хотя все они имеют место.

\section{Список литературы}

1. Repovs D., Semenov P. V. Continuous selections of multivalued mappings. Dordrecht: Kluwer Acad. Publ., 1998.

2. Ahmed N. U. Optimization and identification of systems governed by evolution equations on Banach space. Harlow / New York: Longman Scientific \& Tecnical / John Wiley \& Songs, 1988.

3. Tolstonogov A.A. Differential inclusions in a Banach space. Dordrecht: Kluwer Acad. Publ., 2000.

4. De Blasi F. S., Pianigiani G., Staicu V. Topological properties of nonconvex differential inclusions of evolution type // Nonlinear Anal. 1995. V. 24. P. 711-720.

5. De Blasi F. S., Pianigiani G., Staicu V. On the solution sets of somenonconvex hyperbolic differential inclusions // Czechoslovak Math. J. 1995. V. 120. P. 107-116.

6. Tolstonogov A. A. Continuous selectors of fixed point sets of multifunctions with decomposable values // Set-Valued Anal. 1998. V. 6. P. 129-147. 
7. Толстоногов $A . A . L_{p}$-непрерьвные селекторы неподвижных точек многозначных отображений с разложимыми значениями. III. Приложения // Сиб. матем. журн. 1999. Т. 40. №6. C. $1380-1396$.

8. Fryskowski A., Rzezuchowski T. Continuous version of Filippov-Wazewski relaxation theorem // J. Differential Equations. 1991. V. 94. P. 254-265.

9. Жиков В.В., Козлов С.М., Олейник О.А., Ха Тъен Нгоан. Усреднение и G-сходимость дифференциальных операторов // УМН. 1979. Т. 34. № 5. С. 65-133.

10. Жиков В.В., Козлов С. М., Олейник O.A. О G-сходимости параболических операторов // УМН. 1981. Т. 36. № 1. С. 11-58.

11. Колпаков A. Г. G-сходимость одного класса эволюционных операторов // Сиб. матем. журн. 1988. Т. 29. № 2. С. 90-104.

12. Толстоногов $A . A . L_{p}$-непрерывные селекторы неподвижных точек многозначных отображений с разложимыми значениями. I. Теоремы существования // Сиб. матем. журн. 1999. T. 40. № 3. C. 695-709.

13. Толстоногов $A . A . L_{p}$-непрерывные селекторы неподвижных точек многозначных отображений с разложимыми значениями. II. Теоремы релаксации // Сиб. матем. журн. 1999. T. 40. № 5. C. 1167-1181.

14. Куратовский К. Топология. Т. 2. М.: Мир, 1965.

15. Энгелькинг Р. Общая топология. М.: Мир, 1986.

16. Бурбаки Н. Топологические векторные пространства. М.: ИЛ, 1959.

17. Гаевский $X$., Грегер $K$., Захариас $K$. Нелинейные операторные уравнения и операторные дифференциальные уравнения. М.: Мир, 1978.

18. Papageorgiou N.S. On the "bang-bang" principle for nonlinear evolution inclusions // Dynam. Systems Appl. 1993. V. 2. P. 61-74.

19. Tolstonogov A.A., Tolstonogov D.A. $L_{p \text {-continuous extreme selectors of multifunctions }}$ with decomposable values: existence theorems // Set-Valued Anal. 1996. V. 4. P. 173-203.

20. Hiai F., Umegaki $H$. Integrals, conditional expectations and martingales of multivalued functions // J. Multivariate Anal. 1977. V. 7. P. 149-182.

21. Brezis H. Operateurs maximaux monotones. Amsterdam: North Holland, 1973.

22. Толстоногов A.A. Экстремальные селекторы многозначных отображений и принцип "бэнг-бэнг" для эволюционных включений // Докл. АН СССР. 1991. Т. 317. №3. C. 589-593.

23. Sokolowski J. Optimal control in coefficients for weak variotional problems in Hilbert space // Appl. Math. Optim. 1981. V. 7. P. 283-293.

24. Толстоногов A.A. Свойства множества допустимых пар "траектория-управление" эволюционных управляемых систем первого порядка // Изв. РАН. Сер. матем. 2001. T. 65. №3. C. 201-224.

Институт динамики систем и теории управления СО РАН,

Поступила в редакцию г. Иркутск-33

28.05 .2002 и 20.05 .2003

E-mail: aatol@icc.ru 